\title{
Interference avoidance throughput optimization in cognitive radio ad hoc networks
}

\author{
Nan Hao ${ }^{1}$ and Sang-Jo $\mathrm{Yoo}^{2^{*}}$
}

\begin{abstract}
The development of a method to ensure interference free transmission with various system constraints to achieve maximum secondary network capacity in an underlay cognitive radio (CR) network is still a challenging issue. Due to the lack of explicit support from the primary system, CR sensing algorithms often face difficulties in reliably estimating the primary signals. This problem becomes even worse when the primary user is a pure receiver. In this paper, we propose a distributed neighbor coordinated adaptive power control estimation mechanism to guarantee a reliable protection for primary users. With reliable transmission power control for the secondary transmitter, the power allocation problem of the CR system is modeled into a constrained Lagrange multipliers optimization problem to guarantee reliable sum rate maximization. Based on a comprehensive analysis from the topology and spectrum access scenario, we simplify the complex interference relationship between primary users and CR transmission pairs and propose an iterative power allocation algorithm with a suboptimal bit loading algorithm, along with fully distributed and neighbor coordinated channel selection strategies. Simulation results show that our proposed power spectrum mask estimation algorithm with capacity maximization model can greatly reduce interference to primary nodes and can successfully enhance the overall throughput.
\end{abstract}

Keywords: Cognitive radio, Ad-hoc networks, Optimization, Power loading

\section{Introduction}

Traditional fixed channel allocations result in low efficiency in terms of spectrum utilization. According to the Federal Communication Commission [1], most of the allocated spectrum remains underutilized most of the time. By enabling a secondary user to access the spectrum holes, spectrum utilization can be significantly enhanced by cognitive radio (CR) technology [2]. If the spectrum band is not being used by the primary system or if communications between CR devices do not cause any harmful impact on the performance of the incumbent users, then $\mathrm{CR}$ nodes can adaptively coexist with primary users based on sensing schemes [3-6].

Due to the dynamical changes of primary user (PU) activities on licensed bands, controlling the transmission behaviors of secondary users (SUs) and determining how to allocate the limited licensed spectrum resource to maximize the network capacity is a challenging process. To successfully achieve such an allocation, several studies

\footnotetext{
* Correspondence: sjyoo@inha.ac.kr

${ }^{2}$ Graduate School of Information Technology \& Telecommunication, Inha University, 253 Yonghyun-dong, Incheon, Nam-gu 402-751, Korea Full list of author information is available at the end of the article
}

have considered the dynamic spectrum access (DSA) issues. Especially in orthogonal frequency division multiplexing (OFDM) CR networks, the power allocation is normally formulated as a constrained optimization problem to distribute the available power over several subchannels (SCs) in an optimal way. Such allocation is able to maximize the secondary users' capacity under various constraints [7-12]. In [7], a weighted sum rate for ad hoc SU links in a cognitive radio network was optimized under a power spectrum mask (PSM) of the SU transmitters. In [8], a single cell downlink model was proposed and the weighted sum rate maximization of all PUs and SUs was studied by using sequential quadratic programming and proportional fair scheduling algorithms. In [9], the interference induced by the SU to the PU was considered as a constraint and several suboptimal algorithms were determined. In [10] and [11], it was assumed that the interference limit at the primary receiver is known to SUs based on the assumption that the PU receiver is also a transmitter; these studies proposed an optimization model with several constraints to maximize the CR system capacity. In [12], a novel effective iterative waterfilling algorithm was proposed for power allocation in

\section{然}


cognitive networks with a power budget and the peak transmission power of the SU as constraints. This method introduced a distance model to convert the constraints on the interference induced by SUs to the PUs to the PSM constraint of the SU.

A more complex power spectrum mask fitting proposal was proposed in [13] to allow coexistence between a single cognitive radio cell and multi cell primary cells. The power allocation for subcarriers of the SU was modeled into a constrained optimization problem, in which the mutual interference between the SU and PU was comprehensively formulated as restrictions on the SU's transmission power. Consequently, the modeling scheme restrains the interference to PUs and maximizes the capacity of the SU as well. A similar optimization with a more complete secondary co-channel transmission model is described in [14], in which each channel is shared by multiple SUs. However, [13] and [14], except for the fact that strong interference from the primary transmitter (PT) may interrupt the nearby concurrent secondary communication during the primary uplink phase, ignore the following facts: 1) uplink phase only transmission reduces the spectrum utilization ratio and 2) the primary transmission schedule should always be known in advance, because it will cause unbearable information leaking of primary systems. In [15], an assumption similar to that in [7-14] was used, and the power allocation for the subcarrier of the $\mathrm{SU}$ was modeled into a constrained optimization problem, in which the interference constraint was given as a sensing range concept. With a modified classical power distribution algorithm, the CR network capacity can be maximized with affordable interference to the primary user.

Generally, the power allocation issue for secondary users in cognitive networks includes two aspects: 1) a single user case that considers power allocation for a single SU without a centralized controller or coordinator, in which the accumulated interference from multiple SUs to PUs will not be considered, which is more suitable for point to point transmission; and 2) a multiple SU case that considers the joint power optimization of multiple SUs and accurate interference constraint with various system constraints to help SUs achieve global optimization based on cooperation from a centralized cluster header or coordinator. In this paper, we consider both cases. Despite the contributions of the previous studies, a method that can be used to derive a reliable interference free constraint that plays a key role in capacity maximization has not been well studied in CR ad hoc networks. As a common approach, existing optimization studies in the area of cognitive radio networks assume that the primary transmission range can reach the secondary sensing device that wants to transmit data. In this case, when any primary receiver is within the transmission range of the secondary device, harmful interference (the harmful interference denotes the interference that exceeds the affordable decoding threshold of nearby primary receiver, which is caused by the secondary utilization of the cognitive radio communication pair on the licensed band) higher than the allowed level can be given to the primary receiver. In particular, if the primary receiver does not transmit any signals when the secondary devices perform sensing, then it is very difficult to recognize this primary hidden receiver. In this case, the interference avoidance constraint used for a power spectral mask at the secondary transmitter does not work well. Harmful interference to a primary receiver cannot be avoided. Furthermore, since the Lagrange model has generally been exploited as the main technique in dynamical spectrum allocation optimization for non convex problems with various complicated constraints, the complexity of constraints determines the convergence speed as well as the design difficulty of the optimization algorithm.

Therefore, two main challenges will be raised due to the introduction of a cognitive radio technique into the ad hoc network: 1) how to avoid possible interference to PUs, especially the pure primary receiver (PR), and how to control the accumulated interference to the PR when multiple $C R$ links exist; and 2) how to design maximization criteria along with less complex system constraints with the Lagrange multiplier optimization problem to enhance the $\mathrm{CR}$ system capacity and reduce the calculation complexity.

To solve the problems mentioned above, in this paper, we first propose a distributed neighbor coordinated PSM constraint estimation algorithm to guarantee reliable protection for primary users in a challenging network scenario. If there is reliable transmission power control at the secondary transmitter side, then, in order to maximize the CR network throughput (the maximization of sum of data rates from all sub-channels belongs to $\mathrm{i}$-th $\mathrm{CR}$ communication pair), a Lagrange optimization problem with different channel selection strategies is modeled; in this problem, both fully distributed channel selection and competition based optimal channel selection are considered. Based on a comprehensive analysis using topology and spectrum access scenarios in the proposed network model, the complicated mutual interference between primary users and CR users is simplified to reduce the primary protection uncertainty and the calculation complexity. We jointly considered the relationship among optimal channel set selection, total transmission power budget, and PSM limitation, and propose an efficient iterative power allocation algorithm along with a suboptimal integer bit loading algorithm to optimize the transmission power on the selected channels. 
The remainder of the paper is organized as follows. Section 2 describes the system model and the challenging PSM constraint problem in an ad hoc CR network system. Section 3 provides the proposed neighbor coordinated PSM estimation scheme that provides full protection for primary users even in extreme network scenarios. Section 4 formulates the power requirements to transmit data and the corresponding interference free PSM constraint. A Lagrange multiplier optimization problem is then formulated in Section 5 with simple but efficient power loading algorithms. Section 6 shows the experimental results of the simulations. Section 7 concludes this paper.

\section{PSM constraint problem}

\section{System model}

In this study, a multi-channel transmission scenario for cognitive radio users is assumed. The available spectrum bands are divided into a set of orthogonal channels by using OFDM modulation scheme, so that from the total $\mathrm{N}$ orthogonal sub-channels each cognitive transceiver can opportunistically transmit data using any available sub-channel that does not give harmful interference to primary systems. We assume that the $\mathrm{CR}$ transmitter has perfect knowledge of primary users $\left(P_{\mathrm{PT}}\right.$ and $\left.T R_{\mathrm{PT}}\right)$. $\mathrm{K}$ secondary communication pairs and $\mathrm{M}$ primary communication pairs exist. To avoid harmful interference to primary systems, the CR transmitter needs to sense the existence of primary signals and avoid using those subchannels that do not meet the minimal transmission power needed to maintain the decodable SINR (signal to interference ratio) at the cognitive radio receiver side. The typical PSM estimation model for cognitive radio ad hoc networks [10] is presented in Figure 1. The

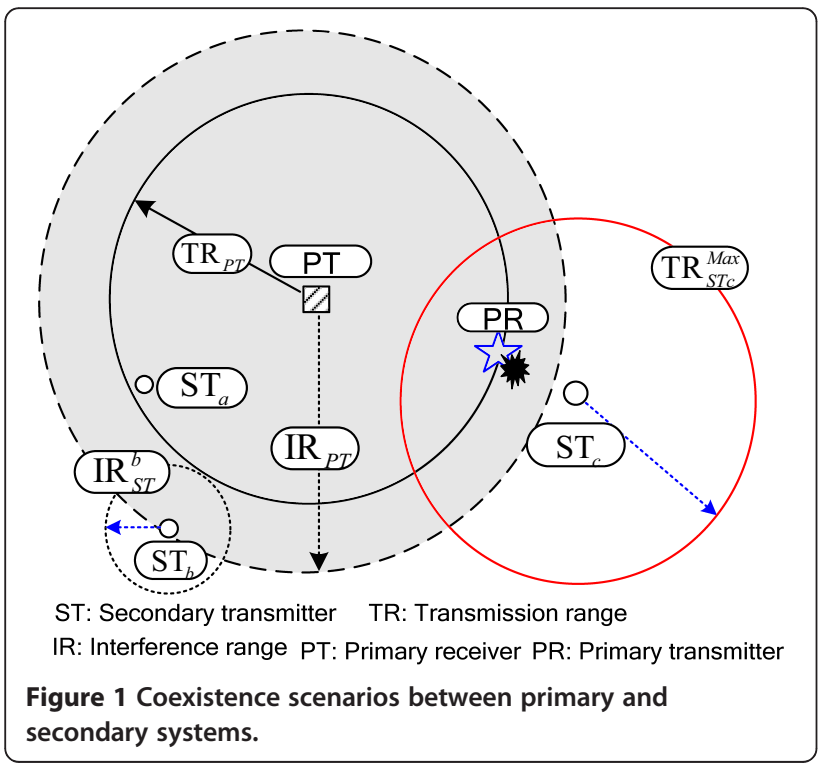

transmission power of the primary transmitter (PT) is $P_{\mathrm{PT}}$; the effective transmission range (i.e., the range decodable by the primary receiver) of the primary system is $T R_{\mathrm{PT}}$; the primary signal interference range is $I R_{\mathrm{PT}}$ ; and the maximal transmission range of secondary transmitter (ST) on the licensed band is $T R_{S T}^{\mathrm{Max}}$ with maximal transmission power. It is assumed that the typical primary transmission power $P_{\mathrm{PT}}$ and the minimal decodable receiving power at the primary receiver are known in advance to the secondary users. It is also assumed that by using a suitable path loss model, the distance between the transmitter and receiver can be derived by measuring the received power for the given transmission power.

\section{Illustration of PSM constraint problem}

Generally, three sensing scenarios exist, as shown in Figure.1: 1) Secondary transmitter a (STa) is within the primary transmission range, which is defined as a protection range in IEEE 802.22 WRAN [16]). STa detects a primary signal, and no transmission is permitted; 2) Secondary transmitter b $(\mathrm{STb})$, which is within the interference range of the PT (also denoted as keep out range in the IEEE 802.22 WRAN [16]), detects the primary signal so that it will control its transmission power to keep the interference range of $\mathrm{STb}\left(I R_{\mathrm{ST}}^{\mathrm{b}}\right)$ outside the protected range of PT; and 3) Secondary transmitter c STc is outside the interference range $I R_{\mathrm{PT}}$ of the primary signal. STc cannot detect the primary signal from PT, so that harmful interference may be given to possible primary receivers (PR) nearby because STc will use the allowed maximal transmission power. In this paper, we consider the case in which the primary receiver does not send any signals when the secondary devices sense the channel. Since a primary receiver does not transmit signals, it is very difficult or impossible for neighboring $\mathrm{CR}$ devices to recognize the existence of such a primary receiver. This situation becomes even worse when STs are within the $I R_{\mathrm{PT}}$, due to the complexity of the wireless transmission environment (e.g., channel fading, shadowing, and signal attenuation) [17]. When the secondary transmitter does not detect nearby primary receivers, it may use the allowed maximum transmission power, and significant inference to those PRs will occur. Especially, when the PR is located close to the ST, this creates the worst case scenario in the field of cognitive radio operation, which aims to protect the primary user. Transmission power control without consideration of hidden primary receivers contradicts the original principle of CR: the implementation of $\mathrm{CR}$ must not impinge on the performance of primary users. Therefore a more reliable power spectral mask estimation scheme for a multi-channel cognitive environment is needed to guarantee the correctness of CR capacity maximization. 


\section{Proposed distributed neighbor coordinated psm estimation}

In this section, we propose a fully distributed low complex neighbor coordinated power spectrum mask constraint estimation method (DNPSM). Each CR transmitter estimates its possible maximum interference range not only based on the primary sensing information gathered directly from itself, but also with the help of one hop secondary neighbors (SNs). Table 1 shows the definitions of the parameters that are used for the distance estimation calculations in this paper.

As can be seen in Figure 2, the secondary transmitter (ST) cannot sense the primary signal because it is not within the primary signal detection range. Therefore, ST itself cannot correctly estimate the distance $D_{P T, S T}$, which is fundamental information for ST power control. To effectively protect primary nodes, the ST must be able to estimate the distance between the ST and the nearest primary receiver (PR). In this paper, we propose a method to estimate the distance $D_{\mathrm{PT} \text {.ST }}$ with the help of secondary neighbor nodes. The control information exchanged to estimate the distance is performed on the CR control band (e.g., the dedicated control channel for CR use or an unlicensed band such as the ISM band), which does not create any harmful interference (Harmful interference denotes interference that exceeds the affordable decoding threshold of a nearby primary receiver, which interference is caused by the secondary utilization of the cognitive radio communication pair on the licensed band) to the primary system working on the licensed band. It should be noted that the ST can use its maximum transmission power on the control channel to contact nearby secondary neighbors. As can be seen in Figure 2, a secondary neighbor (SN) that is located inside the PT's transmission range can overhear the control signal from the ST.
In DNPSM, a secondary neighbor SN can estimate the distance between a CR transmitter and neighbors $\left(D_{\mathrm{ST}, \mathrm{SN}}\right)$ by receiving a message from the secondary transmitter on the control channel; it can also estimate the distance between PT and SN ( $\left.D_{P T, S N}\right)$ by receiving a signal from the primary transmitter on the licensed band. Since SN has already determined the maximum transmission range $D_{P T, P R}$ (e.g., in the case of TV broadcasting) of the primary transmitter, it can estimate the worst case distance $D_{S T, P R}$ (also denoted as safety distance). By reporting the estimated safety distance to ST, ST will control its transmission power so that it does not interfere with the possible primary receiver.

Figure 3 shows the procedure of the proposed DNPSM operation. Before an actual data transmission, the CR transmitter ST first senses the licensed channels. If the ST can find some available channels in which no primary signal can be detected, then it requests assistance from nearby $\mathrm{CR}$ neighbors to verify the correctness of the sensing results using the CR control band interface. Secondary neighbors measure the received power level from the primary transmitter $\left(P_{P R}\right)$ on the channels listed by $S T$. When the primary transmitter's maximum transmission power $\left(P_{\mathrm{PT}}\right)$ is known to the secondary systems, then, by using the path loss model $\left(P L_{L B}(d)\right)$ of the licensed band as in (1), the distance between PT and SN $\left(D_{P T, S N}\right)$ can be derived from (2). In (1), we used the Hata path loss model [18], which is suitable for urban areas, but any path loss model can be applicable to improve the estimation accuracy under different circumstances.

$$
\begin{aligned}
P L_{L B}(d)= & P_{\mathrm{PT}}-P_{P R}=h(69.55+26.16 \log f \\
& \left.-13.82 \log h_{B}-C_{H}+\left[44.9-6.55 \log h_{B}\right] \log d\right)
\end{aligned}
$$

Table 1 Glossary of new terminology

\begin{tabular}{lr}
\hline Parameter & Definition \\
\hline$P_{P R}$ & Received primary signal power level on a licensed band \\
$P_{P T}$ & Primary transmission power on a licensed band \\
$P_{S T}$ & $C R$ transmission power on a licensed band \\
$P_{S R}$ & Received CR signal power on a CR control band \\
$I_{P T}$ & Interference range of primary transmission signal on a licensed band \\
$R_{S T}$ & Interference range of CR transmission signal on a licensed band \\
$T R_{P T}$ & Maximum distance between primary transmitter and primary receiver \\
$D_{P T, S T}$ & Distance between primary transmitter and secondary (CR) transmitter \\
$D_{S T, P R}$ & Distance between secondary (CR) transmitter and primary receiver \\
$D_{P T, S N}$ & Distance between primary transmitter and secondary (CR) neighbor \\
$D_{S T, S N}$ & Distance between CR transmitter and CR neighbor \\
$D_{S N, P R}$ & Distance between secondary (CR) neighbor and primary receiver \\
\hline
\end{tabular}




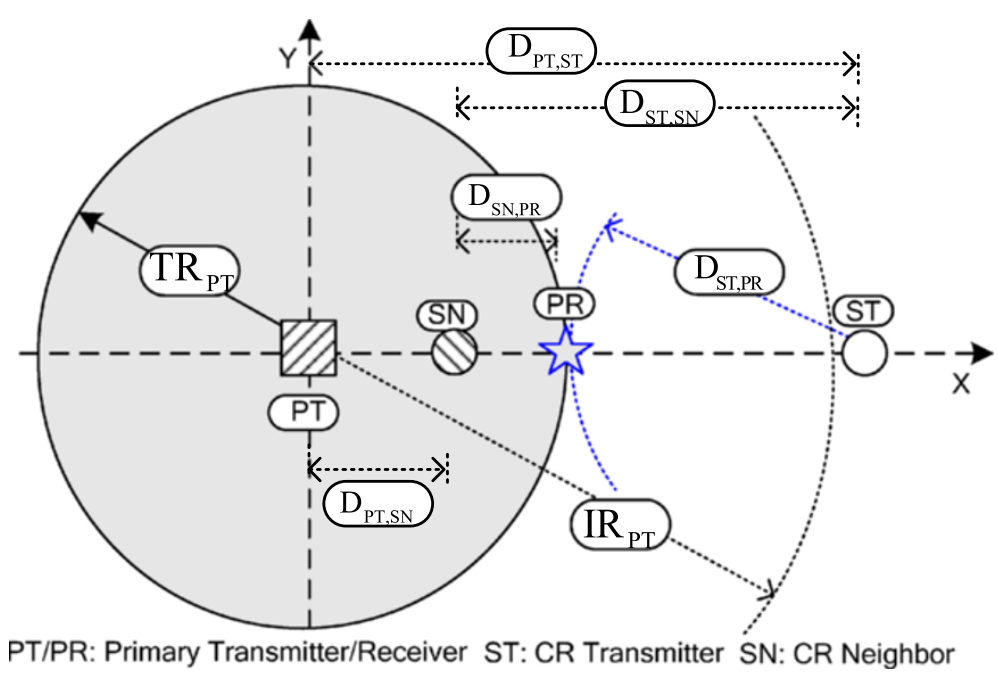

Figure 2 The proposed PSM estimation scheme.

$$
D_{\mathrm{PT}, \mathrm{SN}}=10 \frac{\left[10 \log \left(P_{\mathrm{PT}}-P_{P R}\right)-\left(69.55+26.16 \log f-13.82 \log h_{B}-C_{H}\right)\right]}{\left(44.9-6.55 \log h_{B}\right)}
$$

in which $h_{B}$ is the antenna height of the base station; $C_{H}$ is the antenna height correction factor; $f$ is the transmission frequency; $d$ is the distance between base station and mobile stations; and $h(x)=10^{x / 10}$.

Since the ST transmits the request message on the CR control channel (e.g., a dedicated unlicensed band) with maximal transmission power, some one-hop neighbor $\mathrm{SNs}$ that receive the sensing request may be within the primary transmission range (or interference range). Based on the received power level $P_{\mathrm{SR}}, \mathrm{SN}$ estimates the distance between $\mathrm{ST}$ and $\mathrm{SN}\left(D_{\mathrm{ST}, \mathrm{SN}}\right)$ using a control channel path loss model $\left(P L_{\mathrm{UB}}(d)\right)$. In this paper, we use a typical loss model of the ISM band (COST.231 model [19]).

$$
\begin{gathered}
P L_{\mathrm{UB}}(d)=P_{\mathrm{ST}}-P_{\mathrm{SR}}=h\left(37+30 \log _{10} d+18.3 n^{\left(\frac{n+2}{n+1}-0.46\right)}\right) \\
D_{\mathrm{ST}, \mathrm{SN}}=10^{\frac{10 \log \left(P_{\mathrm{ST}}-P_{\mathrm{SR}}\right)-37-18.3 n}{30}\left(\frac{n+2}{n+1}-0.46\right)}
\end{gathered}
$$

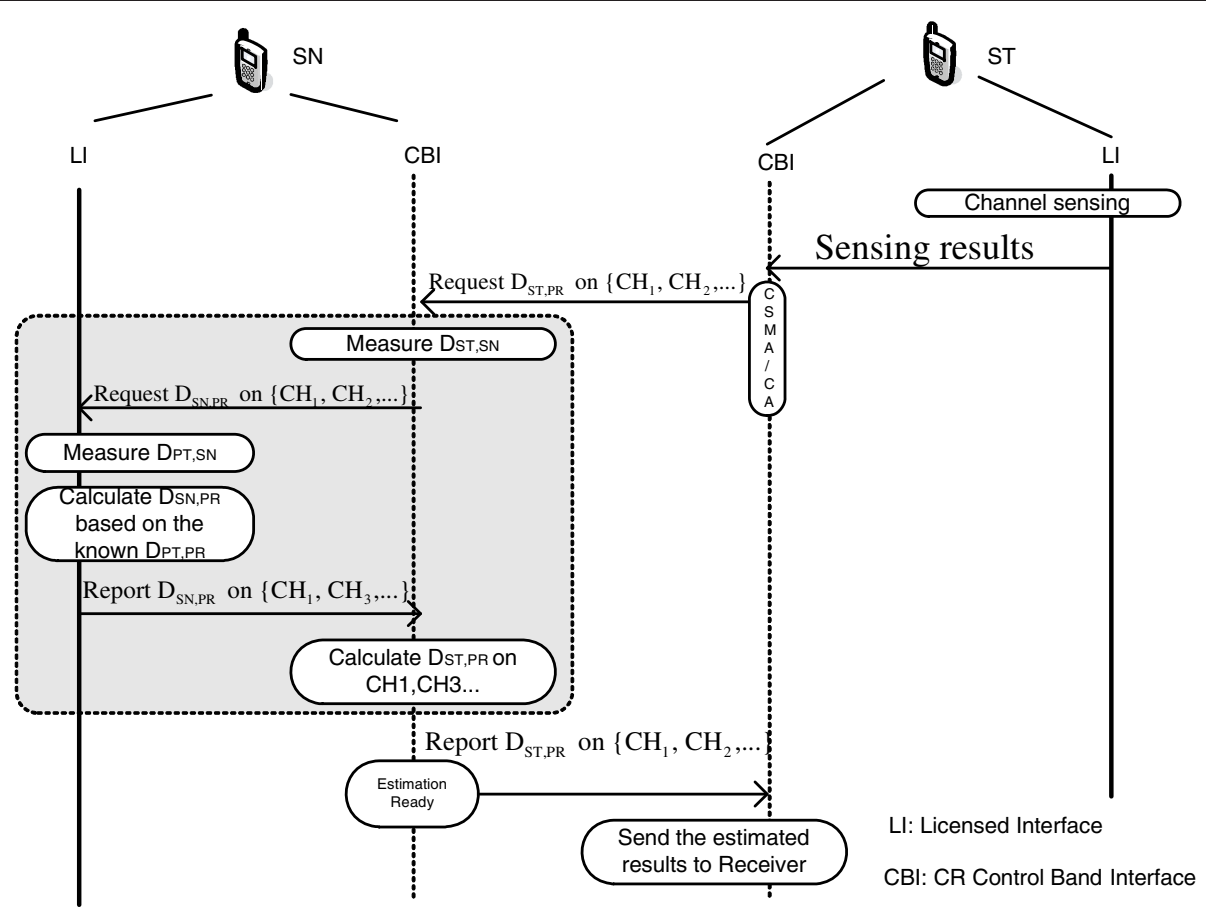

Figure 3 Reliable PSM constraint estimation procedure. 
where $n$ is the path loss parameter that represents the number of floors in the path.

When the maximum primary transmission distance $D_{\mathrm{PT}, \mathrm{PR}}$ is known, the distance $\left(D_{\mathrm{SN}, \mathrm{PR}}\right)$ between $\mathrm{SN}$ and the possible worst case primary receiver location can be derived as in (5).

$$
D_{\mathrm{SN}, \mathrm{PR}}=D_{\mathrm{PT}, \mathrm{PR}}-D_{\mathrm{PT}, \mathrm{SN}}
$$

From (4) and (5), the safe distance for the secondary transmission on each available licensed channel can be derived as in (6).

$$
D_{\mathrm{ST}, \mathrm{PR}}=D_{\mathrm{ST}, \mathrm{SN}}-D_{\mathrm{SN}, \mathrm{PR}}
$$

If there are multiple primary systems working on the same sub-channel $j(j=1,2,3, \ldots, N)$, ST takes only the shortest distance between itself and the nearest worst case primary system receiver as the safety distance (PSM constraint) among all the estimated safety distances on the sub-channel $j$. Clearly, harmful interference to any possible hidden primary receiver from any single CR communication pair can be successfully avoided using the proposed DNPSM.

\section{Power Required and PSM Constraint Formulation Power requirement}

In this study, we consider each sub-channel as a linear time invariant channel; the additive Gaussian noise in each transmission is zero mean with variance $\sigma_{j}^{2} \cdot G_{j}(d)$ is the channel gain coefficient between the secondary transmitter and its dedicated receiver on sub-channel $j$ with distance $d$. The mean power $P_{i, j}$ of ST $i(i=1,2,3, \ldots, N)$ to transmit $b$ bits per symbol in sub-channel $j(j=1,2,3, \ldots$, $N)$ can be calculated as in (7) [20].

$$
P_{i, j}=\frac{\Gamma \sigma_{j}^{2}}{G_{j}(d)}\left(2^{b_{i, j}}-1\right)
$$

The SNR gap $\Gamma$ of each ST can be calculated from (8). The SNR gap $\Gamma$ denotes the difference between theoretical transmitted bits on the channel and the maximal achieved transmitted bits with the selected coding and modulation scheme.

$$
\Gamma=\frac{1}{3}\left[Q^{-1}\left(\frac{R_{e}}{4}\right)\right]^{2} \frac{\gamma}{\kappa}
$$

$\gamma$ is the SNR degradation immunity, which represents the repair capability when facing channel impairment due to an unpredicted situation; the impairment recovery probability is closely related to an extra amount of modulation performance. is $\kappa$ is the coding gain, which depends on what kind of coding scheme is chosen by the current communication pair. $R_{e}$ is the target error probability. $Q^{-1}(\cdot)$ is the inverse of the famous Q- function, which is defined as in (9).

$$
Q(t)=(2 \pi)^{-1 / 2} \int_{t}^{\infty} e^{-\tau^{2} / 2} d \tau
$$

\section{PSM constraint formulation}

The purpose of capacity maximization is to maximize the data transmission rate sum, which is directly related with the $C R$ transmission power on each sub-channel. We assume that the maximal affordable interference power threshold at each primary receiver is the same, and that such a threshold is evenly distributed across all sub-channels with $\zeta$ (the method for obtaining $\zeta$ is given in [13]). Then, the CR transmission power of the ST should be controlled as in (10) to avoid harmful interference to the primary receiver (distance between the ST and PR is $d$ ) within the maximal CR interference range. $I_{j}^{\prime}\left(d^{\prime}\right)$ is the channel gain between the primary transmitter and primary receiver with distance $d^{\prime}$. $T P_{\mathrm{PT}} I_{j}^{\prime}\left(d^{\prime}\right)$ is the received power level from the primary transmitter working on channel $j$ at the primary receiver with distance $d^{\prime}$.

$$
P_{i, j} G_{j}(d) /\left(\delta_{j}^{2}+T P_{\mathrm{PT}} I_{j}^{\prime}\left(d^{\prime}\right)\right) \leq \zeta
$$

By manipulating (1), $G_{j}$ and $I_{j}^{\prime}$ can be obtained by (11) and (12).

$$
\begin{aligned}
& G_{J}\left(D_{\mathrm{ST}, \mathrm{PR}}\right)=10^{-0.1 P L_{\mathrm{LB}}\left(D_{\mathrm{ST}, \mathrm{PR}}\right)} \\
& I_{j}^{\prime}\left(T R_{\mathrm{PT}}\right)=10^{-0.1 P L_{\mathrm{LB}}\left(T R_{\mathrm{PT}}\right)}
\end{aligned}
$$

Then, (10) can be rewritten as in (13), combining (11) and (12).

$$
P_{i, j} \leq \zeta\left(\left(\delta_{j}^{2}+T P_{\mathrm{PT}} 10^{-0.1 P L_{\mathrm{LB}}\left(T R_{\mathrm{PT}}\right)}\right)\right) 10^{0.1 P L_{\mathrm{LB}}\left(D_{\mathrm{ST}, \mathrm{pR}}\right)}
$$

Let us assume that $\mathrm{TP}_{\mathrm{ST}}^{\mathrm{Max}}$ is the maximal allowed transmission power limited by the CR system hardware on each sub-channel. Then, to avoid harmful interference to primary system receivers, and based on the DNPSM and by manipulating (1) according to the estimated $D_{\mathrm{ST}, \mathrm{PR}}$, as shown in Figure 4 , the maximal allowed transmission power $\left(\mathrm{P}_{\mathrm{ST}}^{\mathrm{Max}}\right)$ of the secondary transmitter is given in (14). $I R_{S T}^{\operatorname{Max}}$ is the interference range of $C R$ 


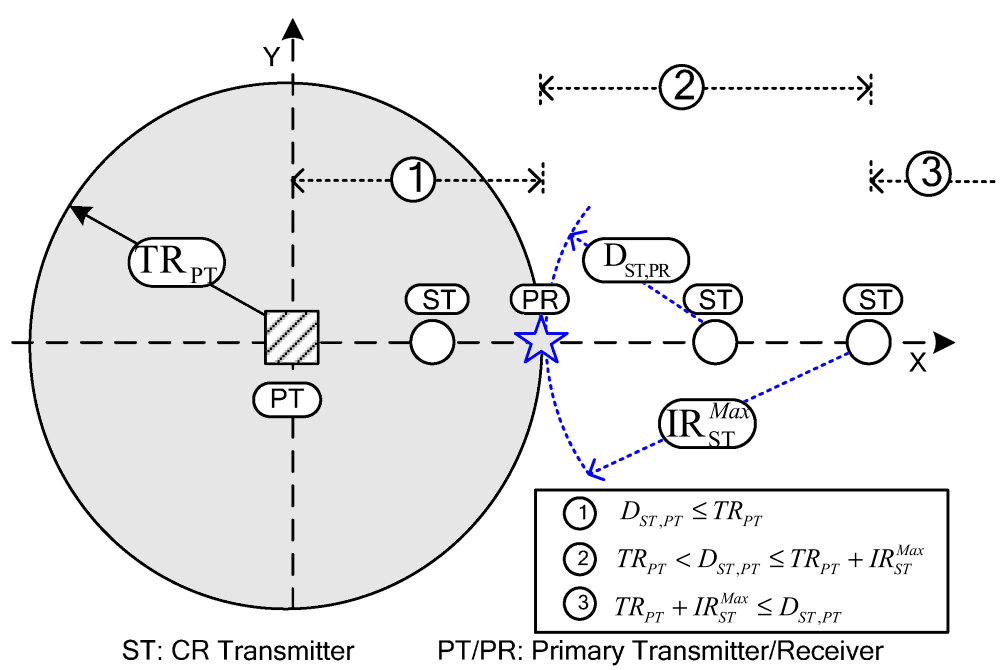

Figure 4 Illustration of PSM constraint with distance variation.

transmission using $\mathrm{TP}_{\mathrm{ST}}^{\mathrm{Max}} . P_{i, j}$ is the transmission power of ST i on sub-channel $j$.
With the total energy amount as the power budget limitation, and the PSM constraint as the maximal trans-

$P_{i, j} \leq P_{\mathrm{ST}}^{\mathrm{Max}}= \begin{cases}0, & D_{\mathrm{ST}, \mathrm{PT}} \leq \mathrm{TR}_{\mathrm{PT}} \\ \zeta\left(\left(\delta_{j}^{2}+\mathrm{TP}_{\mathrm{PT}} 10^{-0.1 P L_{\mathrm{LB}}\left(T R_{\mathrm{PT}}\right)}\right)\right) 10^{0.1 P L_{\mathrm{LB}}\left(D_{\mathrm{ST}, \mathrm{PR}}\right)}, & \mathrm{TR}_{\mathrm{PT}}<D_{\mathrm{ST}, \mathrm{PT}} \leq \mathrm{TR}_{\mathrm{PT}}+\mathrm{IR}_{\mathrm{ST}}^{\mathrm{Max}} \\ \mathrm{TP}_{\mathrm{ST}}^{\mathrm{Max}}, & \mathrm{TR}_{\mathrm{PT}}+\mathrm{IR}_{\mathrm{ST}}^{\mathrm{Max}} \leq D_{\mathrm{ST}, \mathrm{PT}}\end{cases}$
With transmission power $P_{i, j}$ from (14), harmful interference from a single ST to the nearest PR can be successfully avoided. However, such transmission power results in a nutritious accumulated interference from multiple single STs at the same PR side, which is a challenging task that cannot be avoided easily. The solution of this issue will be discussed in the following sections.

\section{Capacity maximization formulation}

\section{Distributed channel selection strategy}

In this part, we consider a fully distributed network in which each CR communication pair performs the CSMA/CA scheme defined in IEEE 802.11 WLAN to obtain the right to spectrum access. The communication pair will utilize all available sub-channels to transmit data. Since we have multiple CR communication pairs on sub-channel $j(j=1,2,3, \ldots, N)$, if there are $K$ active CR transmissions on the same channel $j$, then, harmful interference caused by a single CR communication pair (10) should be rewritten as an accumulative interference constraint (15) with each $P_{i, j}$ following (14). $D_{S T i, \mathrm{PR}}^{j}$ is the distance between ST $i(i=1,2,3, \ldots, K)$ and the nearest PR on sub-channel $j$.

$$
\sum_{i=1}^{K} P_{i, j} G_{j}\left(D_{S T i, \mathrm{PR}}^{j}\right) /\left(\delta_{j}^{2}+T P_{\mathrm{PT}} I_{j}^{\prime}\left(T R_{\mathrm{PT}}\right)\right) \leq \zeta
$$

mission power, the capacity maximization problem can be formulated as a Lagrange multiplier optimization problem on $N$ sub channels; this problem is given in (16), in which $d_{i, m}$ is the distance between the $i$ th CR communication pair and the primary transmitter $m(\mathrm{~m}=1$, $2,3, \ldots, M)$, and $D_{\mathrm{ST} k \text { SR } i}$ is the distance between the ST $k$ ( $k \neq i, k=1,2,3, \ldots, K)$ and the dedicated receiver of $i$ th $\mathrm{CR}$ communication pair.

$$
\begin{aligned}
& \operatorname{Max} \sum_{j=1}^{N} \log _{2}\left(1+P_{i, j} G_{j}\left(D_{\mathrm{ST} i, \mathrm{SR} i}\right) /\left(\delta_{j}^{2}+\sum_{m \in M} T P_{\mathrm{PT}} I_{j}^{\prime}\left(d_{i, m}\right)\right.\right. \\
& \left.\left.\quad+\sum_{\substack{k=1 \\
k \neq \mathrm{i}}}^{K} P_{k, j} G_{j}\left(D_{S T k, S R i}\right)\right)\right)
\end{aligned}
$$

subject to:

$$
\sum_{j=1}^{N} P_{i, j}-P_{\text {Budget }} \leq 0
$$

$$
\begin{gathered}
\sum_{i=1}^{K} P_{i, j} G_{j}\left(D_{S T i, \mathrm{PR}}^{j}\right) /\left(\delta_{j}^{2}+T P_{\mathrm{PT}} I_{j}^{\prime}\left(T R_{\mathrm{PT}}\right)\right) \leq \zeta \\
P_{i, j}^{\mathrm{Min}} \leq P_{i, j}
\end{gathered}
$$

$P_{i, j}^{\mathrm{Min}}$ is the minimal required transmission power of ST $i$ to its dedicated receiver SR on channel $j$. Because the 
received SINR at the secondary receiver should be greater than the minimum signal decodable level $\gamma_{S}$ on each channel $j$, the minimum transmission power $P_{i, j}^{\mathrm{Min}}$ of ST $i$ on channel $j$ can be obtained from (17).

$$
\gamma_{s} \leq \frac{P_{i, j}^{\mathrm{Min}} G_{j}\left(D_{\mathrm{ST} i, \mathrm{SR} i}\right)}{\delta_{j}^{2}+\sum_{m \in M} T P_{\mathrm{PT}} I_{j}^{\prime}\left(d_{i, m}\right)+\sum_{\substack{k=1 \\ k \neq \mathrm{i}}}^{K} P_{k, j} G_{j}\left(D_{\mathrm{ST} k, \mathrm{SR} i}\right)}
$$

\section{Analysis of the capacity maximization model}

To control the harmful accumulated interference caused by $\mathrm{CR}$ transmissions to the primary receiver under the affordable threshold (15), the ST needs to know the number, transmission power, and channel gain of all neighboring co-channel CR transmission pairs. This is a challenging task and many studies consider just a single secondary communication pair instead [7-12]. In some investigations, the spectrum access of the $\mathrm{SU}$ in a multiple SU network model follows the exclusively spectrum access (ESA) model, in which only one CR communication pair is allowed each time, in order to avoid accumulated interference from the secondary transmission to the primary receiver on the same channel [13][14]. In this study, we consider a multiple CR communication pairs model without the ESA limitation; in this model, multiple secondary users are able to access the same subchannel by performing the CSMA/CA scheme. A topology based interference relationship analysis between multiple STs and the same PR is shown in Figure 5.
Considering Figure 5, let us suppose that $D_{\mathrm{ST} a, \mathrm{PR} a}^{\mathrm{SC}_{1}}$ is the CR ST $a$ ' estimated safety distance to the nearest PR (PRa) working on sub-channel $\mathrm{SC} 1$ by performing DNPSM, and $D_{\mathrm{ST} b, \mathrm{PR} b}^{\mathrm{SC}_{1}}$ is the CR ST $b^{\prime}$ estimated safety distance to the nearest $\mathrm{PR}(\mathrm{PRb})$ working on SC1. Both of these are the shortest distances to the nearby PRs (PRa and $\mathrm{PRb}$ ) on the sub-channel SC1. If the STa gives harmful interference to the $\mathrm{ST} b$ ' nearest $\mathrm{PRb}$, then $D_{\mathrm{ST} a, \mathrm{PR} a}^{\mathrm{SC}_{1}}$ is not the shortest distance to the nearby PR on channel $j$ around the STa. This contradicts the fact that the estimated $D_{\mathrm{ST} i, \mathrm{PR}}^{j}$ should be the shortest distance from ST $i$ to all PRs around channel $j$. Therefore, with the help of the proposed DNPSM, the existing challenge of accumulated interference to the same primary receiver in a multiple nodes network model can be successfully solved. Besides, the proposed DNPSM assumes that each CR device is equipped with two interfaces: i) the CR control band interface, which is used for sensing information distribution among CR nodes and for exchanging control information; and ii) the licensed band interface (e.g., the UHF band), which is not only used for data transmission, but also for safety PSM constraint derivation between possible primary receiver and CR devices. Due to the merit of having two interfaces, the interference component $P_{k, j} G_{j}\left(D_{\mathrm{ST} k, \mathrm{SR} i}\right)$ of (16) can be released. The reason for this is that all the control information and sensing messages are exchanged on the common control channel through CSMA/CA, which is defined in IEEE 802.11 WLAN. One-hop neighbors will always overhear the network allocation vector (NAV) of the selected subchannel $j$ from the current $\mathrm{CR}$ communication pair on

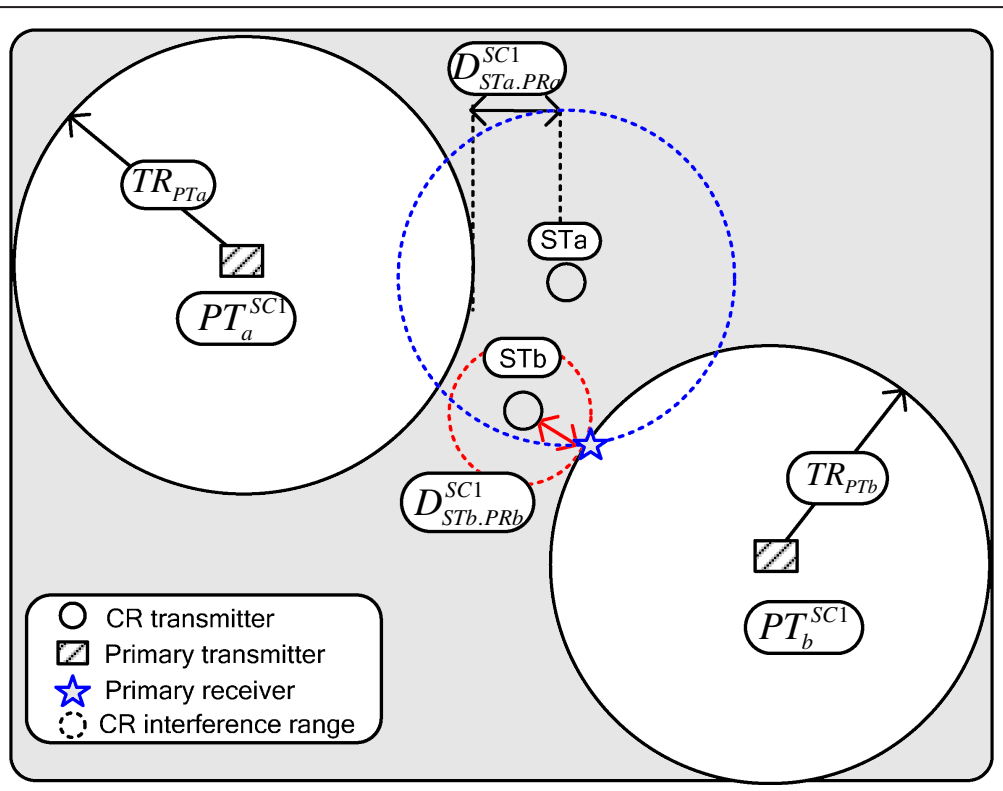

Figure 5 Accumulated interference cancellation illustration. 
the common control channel. The NAV will cease the concurrent transmissions from one-hop CR neighbors. Co-channel interference from two-hop neighboring CR nodes is not considered. Therefore, the complex accumulated interference caused by co-channel CR transmissions can be converted into a single CR communication pair, which simplifies the assumption. Then, (16) can be further simplified and rewritten as (18), in which $P_{i, j}^{\mathrm{Max}}$ is the maximal transmission power of ST $i$ on sub-channel $j$ and is equal to $P_{\mathrm{ST}}^{\mathrm{Max}}$ in (14), with safety distance $D_{\mathrm{ST} i \mathrm{PR}}^{j}$.

$$
\begin{aligned}
\operatorname{Max} \sum_{j=1}^{N} & \log _{2}\left(1+P_{i, j} G_{j}\left(D_{S T i, S R i}\right) /\right. \\
& \left.\left(\delta_{j}^{2}+\sum_{m \in M} T P_{\mathrm{PT}} I_{j}^{\prime}\left(d_{i, m}\right)\right)\right)
\end{aligned}
$$

subject to :

$$
\begin{gathered}
\sum_{j=1}^{N} P_{i, j}-P_{\text {Budget }} \leq 0 \\
P_{i, j} \leq P_{i, j}^{\mathrm{Max}} \\
P_{i, j}^{\mathrm{Min}} \leq P_{i, j}
\end{gathered}
$$

\section{Local power loading algorithm}

By using the Karush-Kuhn-Tucker (KKT) condition [21], and applying the Lagrange multiplier approach, the maximization power filling solution of (18) can be obtained by deriving (19) with $\lambda \geq 0, \mu_{i} \geq 0$.

$$
\begin{gathered}
\frac{G_{j}\left(D_{\mathrm{ST} i, \mathrm{SR} i}\right) /\left(\delta_{j}^{2}+\sum_{m \in M} T P_{\mathrm{PT}} I_{j}^{\prime}\left(d_{i, m}\right)\right)}{\ln 2 \times\left(1+P_{i, j} G_{j}\left(D_{\mathrm{ST} i, \mathrm{SR} i}\right) /\left(\left(\delta_{j}^{2}+\sum_{m \in M} T P_{\mathrm{PT}} I_{j}^{\prime}\left(d_{i, m}\right)\right)\right)\right)} \\
-\lambda-\mu_{i}=0 \\
\lambda\left(\sum_{j \in N} P_{i, j}-P_{\text {Budget }} \leq 0\right)=0 \\
\mu_{i}\left(P_{i, j}-P_{i, j}^{\mathrm{Max}}\right)=0, \forall j \in N
\end{gathered}
$$

In order to reduce complexity of the following algorithm $P_{i, j} \geq P_{i, j}^{\mathrm{Min}}$ is included in the boundary check instead of being included in the Lagrange multiplier problem. By manipulating (19), the optimization filling power $P_{i, j}^{\mathrm{Opt}}$ on channel $j$, necessary to maximize the transmission data rate, can be calculated as in (20).
With the estimated PSM constraint for ST $i$ $(\mathrm{i}=1,2,3, \ldots, \mathrm{K})$ from DNPSM, we know the maximal allowed transmission power $\left(P_{i, j}^{\mathrm{Max}}\right)$. By combining $\lambda$ and $\mu_{i}$ and defining $1 /\left[\ln 2\left(\lambda+\mu_{i}\right)\right]=\varphi_{i}$ as the power loading (water-filling (WL)) level needed to pour limited power into the sub-channel $j(j \in N)$, the water level can be uniquely determined from a total power budget average concept, in which $\varphi_{i}$ can be obtained from derivation by making (21) equal to zero with $N_{i, j}^{-1}$ as given in (20).

$$
f\left(\varphi_{i}\right)=\sum_{j=1}^{N} \operatorname{Max}\left[\left(\varphi_{i}-N_{i, j}^{-1}\right), 0\right]-P_{\text {Budget }}
$$

The only variable parameter in (21) is $\varphi_{i}$, with the measured bottom $\left(N_{i, j}^{-1}\right)$ of each channel and the initial power level $\varphi_{i}$ without PSM constraint able to be simply estimated as in (22).

$$
\varphi_{i}=\left(\sum_{j=1}^{N} N_{i, j}^{-1}+P_{\text {Budget }}\right) / N
$$

Since different sub-channels have different covers $\left(N_{i, j}^{-1}+P_{i, j}^{\mathrm{Max}}\right)$, three kinds of sub-channel exist: 1) maximal loaded sub-channels, in which the allocated amount of power is $\left.\left(P_{i, j}^{\mathrm{Max}}\right) ; 2\right)$ channels in which the power loading is restrained by the water level $\varphi_{i}$, in which the allowed pouring power is $\varphi_{i}-N_{i, j}^{-1}$; and 3) zero power sub-channels, in which no transmission will be allowed on these channels. With the limited power budget, if we pour all sub-channels with $P_{i, j}^{\mathrm{Max}}$, power budget will be exhausted. In order to allow sub-channels in both case 1) and case 2) to be assigned optimal power from a limited power budget, the only method is to take some power from the sub-channels of case 1) and pour it into the sub-channels of case 2). To decide which channel should be selected for the pouring of water and to determine the amount of power to pour on the selected sub-channel in order to maximize the capacity of the CR communication pair, we propose an iterative interference avoidance local rate sum (IALRS) algorithm (Algorithm 1) to derive the optimal value $\varphi_{i}^{\text {Opt }}$ with suitable channel selection. The proposed sub-channel allocation algorithm operates with the following steps. An example of the power-

$$
P_{i, j}^{\mathrm{Opt}}=\left\{\begin{array}{c}
P_{i, j}^{\mathrm{Max}}, \sum_{j \in N} P_{i, j}^{\mathrm{Max}}-P_{\text {Budget }} \leq 0 \\
\operatorname{Min}\left\{\operatorname{Max}\left\{\frac{1}{\ln 2 \times\left(\lambda+\mu_{i}\right)} \varphi_{i}-\left[G_{j}\left(D_{\mathrm{ST} i, \mathrm{SR} i}\right) /\left(\left(\delta_{j}^{2}+\sum_{m \in M} T P_{\mathrm{PT}} I_{j}^{\prime}\left(d_{i, m}\right)\right)\right) N_{i, j}\right]^{-1}, 0\right\}, P_{i, j}^{\mathrm{Max}}\right\}, \sum_{j \in N} P_{i, j}^{\mathrm{Max}}-P_{\text {Budget }}>0
\end{array}\right.
$$


loading scenario is shown in Figure 6. The pseudo code of IALRS is given in Figure 7.

$\left.1^{\text {st}}\right)$ Initial power level estimation: average the total power amount on sub-channel $j(j=1,2,3, \ldots, N)$ according to (22) as the initial water level $\varphi_{i}$.

$\left.2^{\text {nd }}\right)$ Adjustment of power allocation: for sub-channel $j$ $(j \in N)$, check the maximal allowed transmission power (PSM constraint $P_{i, j}^{\mathrm{Max}}$ estimated from (14)), and calculate the minimal required transmission power $\left(P_{i, j}^{\mathrm{Min}}\right)$ using (17). If current water level $\varphi_{i}$ exceeds the cover $\left(N_{i, j}^{-1}+\right.$ $P_{i, j}^{\mathrm{Max}}$ ) of channel $j$, remove current channel $j$, save the power $\varphi_{i}-\left(N_{i, j}^{-1}+P_{i, j}^{\mathrm{Max}}\right)$ in the total power budget $P_{\text {Budget }}$; the number of removed channels is increased by one. If current water level does not cover the noise bottom $\left(N_{i, j}^{-1}+P_{i, j}^{\mathrm{Min}}\right)$ of channel $j$, save the power $\varphi_{i}-N_{i, j}^{-1}$ in the total power budget $P_{\text {Budget }}$; no power will be allocated on this channel, or else $\varphi_{i}-N_{i, j}^{-1}$ should be poured into channel $j$.

$\left.3^{\text {rd }}\right)$ Re-adjust water level: for remaining sub-channels $(N-R)$, average the power budget saved from $2^{\text {nd }}$ step. The new power loading level $\varphi_{i}^{\prime}$ should be within the range of (23), in which $P_{\text {Budget }}^{\mathrm{Left}_{R}}$ denotes the saved power collected from removed channel set $(\mathrm{R})$ due to the effect of the PSM constraint ( $\left.P_{i, j}^{\mathrm{Max}}\right) ; \quad P_{\text {Budget }}^{\mathrm{Left}_{T} R}$ denotes the remaining power collected from the temporary removed (TR) channels whose noise bottoms are not covered by the current power loading level $\left(\varphi_{i}\right) .|x|$ represents the number of sub-channels in the removed channel set $x$.
By defining $\underline{\varpi}=\varphi_{i}+P_{\text {Budget }}^{\mathrm{Left} R} /(N-|R|)$ and $\quad \bar{\varpi}=$ $\varphi_{i}+\left(P_{\text {Budget }}^{\mathrm{Left} R}+P_{\text {Budget }}^{\mathrm{Left} T R}\right) /(N-|R|-|T R|), \quad$ the new water level can be updated as in (24).

$$
\begin{aligned}
\varphi_{i}+ & P_{\text {Budget }}^{\mathrm{Left} \_R} /(N-|R|) \leq \varphi_{i}^{\prime} \leq \varphi_{i}+\left(P_{\text {Budget }}^{\mathrm{Left} R}+P_{\text {Budget }}^{\mathrm{Left} / T R}\right) / \\
& (N-|R|-|T R|) \\
\varphi_{i}^{\prime} & =\frac{\underline{w}+\bar{\varpi}}{2}
\end{aligned}
$$

If $f\left(\varphi_{i}^{\prime}\right)$ in (21) is larger than zero, repeat Step 2 to find lower bound $\underline{\varpi}$, set the upper bound as $\bar{\varpi}=\varphi_{i}^{\prime}$ and repeat Step 3 to find a solution to enable (21) to equal zero. If $f\left(\varphi_{i}^{\prime}\right)$ in (21) is smaller than zero, set up the lower bound as $\underline{\varpi}=\varphi_{i}^{\prime}$, repeat Step 3 to find the updated upper bound $\bar{\varpi}$.

$\left.4^{\text {th }}\right)$ Repeat Steps 2 and 3 until no power can be collected from the previous round; $\varphi_{i}^{\mathrm{Opt}}$ can be obtained $\left(\varphi_{i}^{\mathrm{Opt}}=\varphi_{i}^{\prime}\right.$ in Figure 6).

It should be noted that our algorithm does not need to sort all the sub-channels according to $N_{i, j}^{-1}$, which sorting is an important component of traditional algorithms and is considerably complex.

The iteration performance of the proposed IALRS algorithm is illustrated in Figure 8 , in which the total power budget $\left(P_{\text {budget }}\right)$ equals $800(\mathrm{~dB})$, with noise bottoms and covers randomized from $(0,50)(\mathrm{dB})$ and $(51$, $150)(\mathrm{dB})$, respectively. When we increase the number of

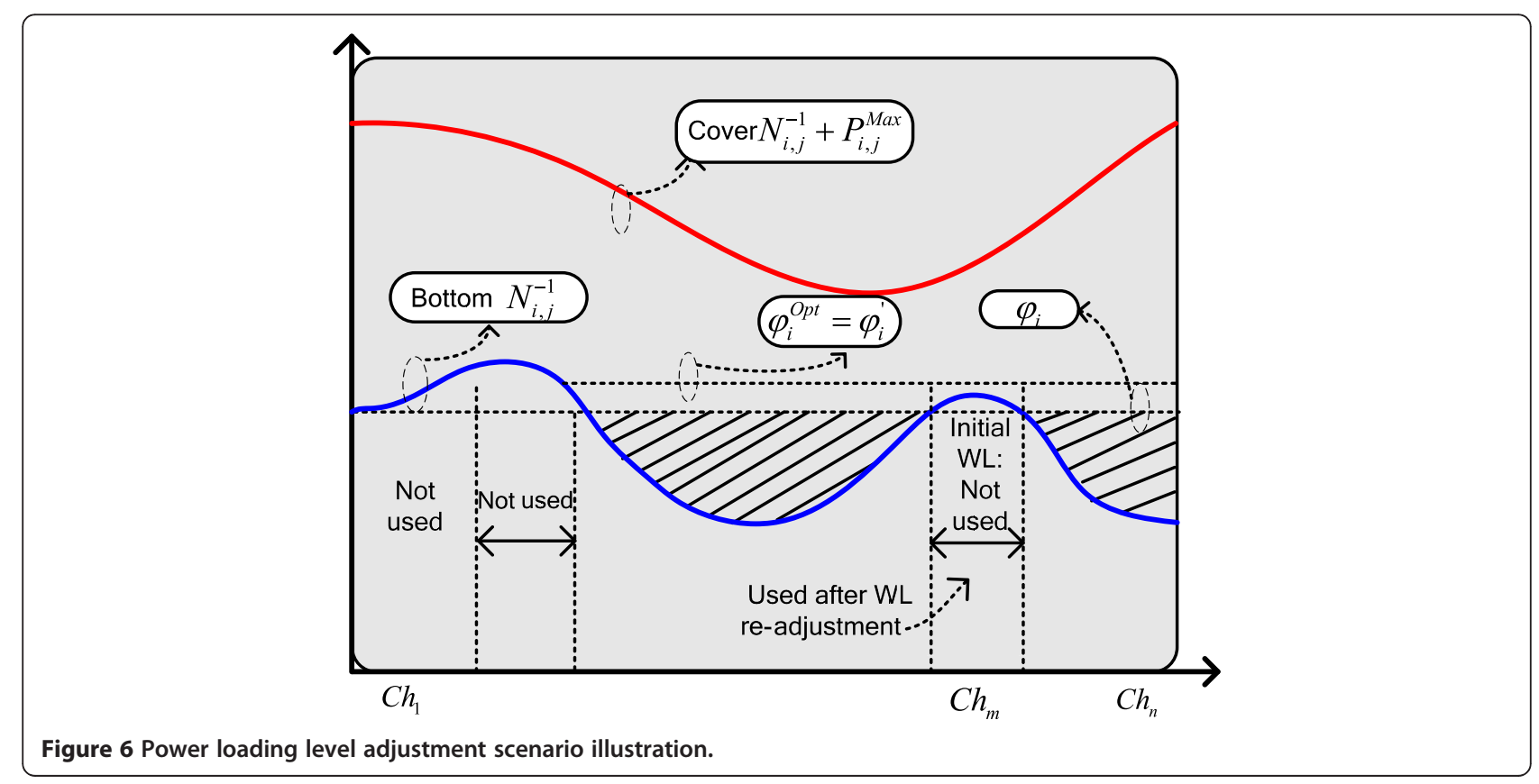




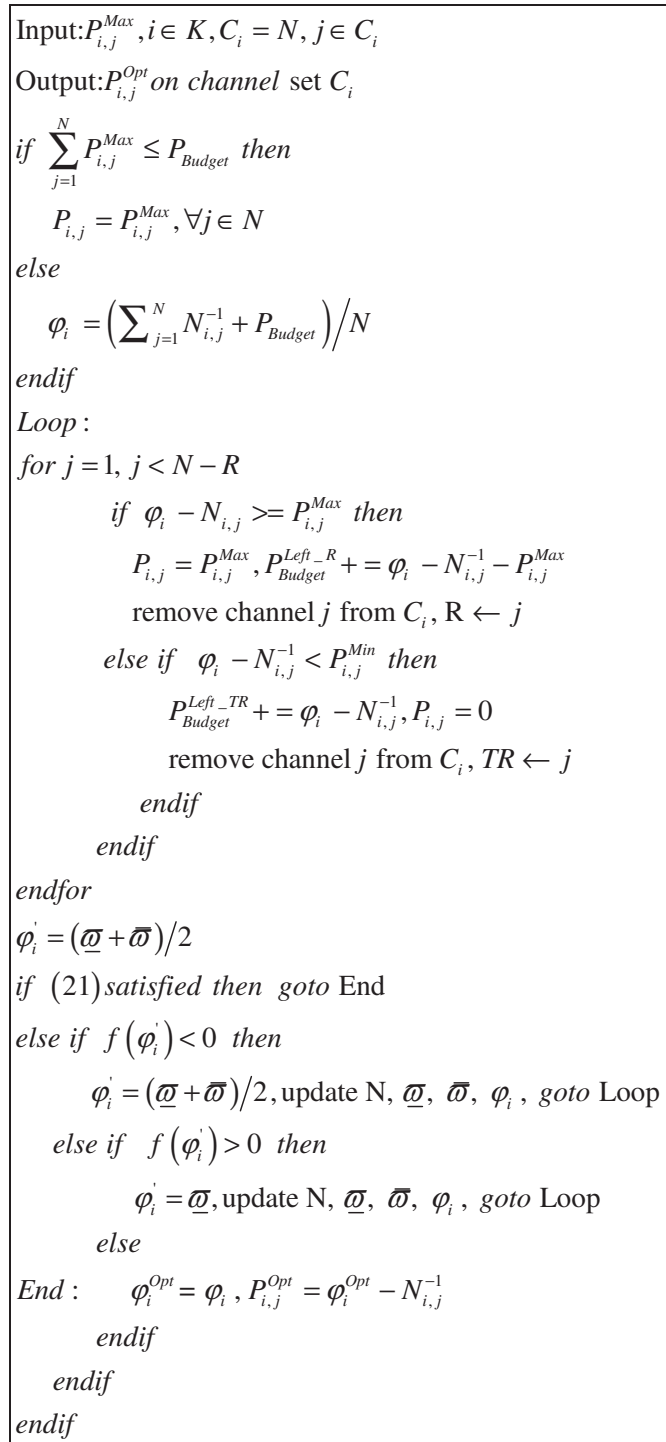

Figure 7 Iterative interference avoidance local rate sum (IALRS) algorithm.

sub-channels, the iteration numbers increase as well. The reason for this is that the number of iterations of the proposed algorithm depends on the number of channels that have been detected that do not satisfy the conditions among the three channel condition criteria $\left(P_{i, j}^{\mathrm{Min}}, P_{i, j}^{\mathrm{Max}}\right.$ and $N_{i, j}^{-1}$ ), which results in a water level readjustment. The fewer the number of channels detected compared to the total number of sub-channels $\mathrm{N}$, the higher the improvement of the computational complexity.

\section{Cooperative Channel Selection Strategy}

In this part, instead of only maximizing the throughput of each CR communication pair separately by using the distributed channel selection strategy, we also take

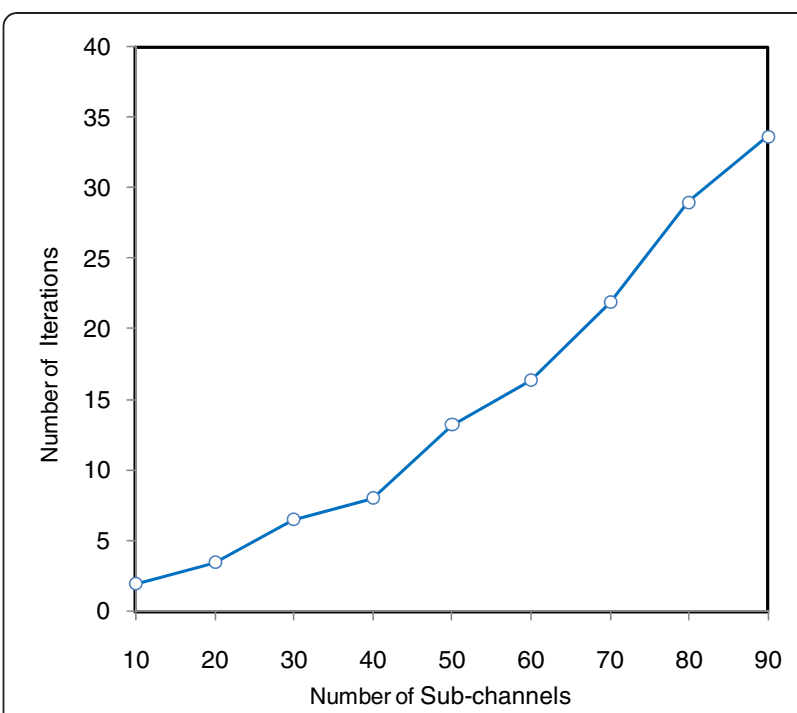

Figure 8 Iterative trend of the proposed interference avoidance local rate sum (IALRS) algorithm.

the cooperative channel selection strategy into consideration, in which CR nodes coordinate with each other and exchange safety distance information among themselves. CR nodes compare their estimated PSM constraint related distance $D_{\mathrm{ST} i, \mathrm{PR}}^{j}$; only ST $i(i=1,2,3, \ldots K)$ with $\arg \underset{i \in K}{\operatorname{Max}}\left(\arg \underset{j \in N}{\operatorname{Min}}\left(D_{\mathrm{ST} i, \mathrm{PR}}^{j}\right)\right)$ wins the spectrum assessing right of channel $j(j=1,2,3, \ldots N)$. It is assumed that the safety distance information among CR nodes is perfectly exchanged. At the end of the channel comparison stage, each CR node has its own channel selection strategy to maximize the CR system capacity (ot only single communication pairs). To maximize the multiple nodes CR network system, the capacity maximization problem (18) can be rewritten as in (25), in which Opt $C_{i}$ is the optimal channel set selected by ST $i$ according to the channel selection criteria.

$$
\begin{gathered}
\operatorname{Max} \sum_{i=1}^{K} \sum_{j \in \mathrm{Opt} C_{i}} \log _{2}\left(1+P_{i, j} G_{j}\left(D_{\mathrm{ST} i, \mathrm{SR} i}\right) /\right. \\
\left.\left(\delta_{j}^{2}+\sum_{m \in M} T P_{\mathrm{PT}} I_{j}^{\prime}\left(d_{i, m}\right)\right)\right) \\
\sum_{j=1}^{\text {OptC } C_{i}} P_{i, j}-P_{\text {Budget }} \leq \\
P_{i, j} \leq P_{i, j}^{\mathrm{Max}} \\
P_{i, j}^{\mathrm{Min}} \leq P_{i, j}
\end{gathered}
$$

Since the optimal sub-channel setOpt $C_{i}$ is selected by each CR communication pair before Lagrange formulation, the optimal transmission power on each optimal channel set can be obtained by the similar procedure as 


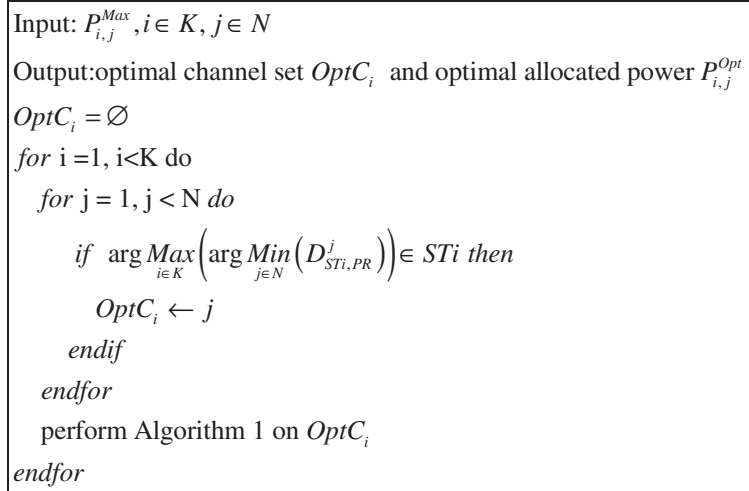

Figure 9 Interference avoidance optimal rate sum (IAORS) algorithm (Algorithm 2).

(19) and (20) of Algorithm 1. The Pseudocode of interference avoidance optimal rate sum (IAORS) solution, which handles the problem of how to find a suitable channel set $\mathrm{OptC}_{i}$ and the suitable power on channel $j$ within each $\operatorname{Opt}_{i}(i=1,2,3, \ldots K)$, are given in Figure 9 (Algorithm 2), with the following operation steps:

$\left.1^{\text {st }}\right)$ Optimal sub-channel set selection: calculate the maximal transmission power of CR node $i(i \in K)$ using (14) on sub-channel $j(j \in N)$ by performing DNPSM; assign the sub-channel $j(j \in N)$ with $\arg \underset{j \in N}{\operatorname{Min}}\left(D_{\mathrm{ST} i, \mathrm{PR}}^{j}\right)$ as the transmission power "covers" for CR node $i(i \in K)$.

$\left.2^{\text {nd }}\right)$ Compare all one hop CR nodes $k(i, k \in K, i \neq k)$ with $\quad \underset{i \in K}{\operatorname{Max}}\left(\arg \underset{j \in N}{\operatorname{Min}}\left(D_{\mathrm{ST} i, \mathrm{PR}}^{j}\right)\right)$.

$\arg \underset{i \in K}{\operatorname{Max}}\left(\arg \underset{j \in N}{\operatorname{Min}}\left(D_{\mathrm{ST} i, \mathrm{PR}}^{j}\right)\right) \in \mathrm{ST} i$, assign the subchannel $j(j \in N)$ to CR node $i(i \in K)$. Repeat $1^{\text {st }}$ and $2^{\text {nd }}$ steps until sub-channels $j(j \in N)$ are assigned to optimal sub-channel set of each CR node $i(i \in K)$.

$\left.3^{\text {rd }}\right)$ Throughput maximization phase: perform Algorithm 1 on $\operatorname{Opt} C_{i}(i \in K)$.

Due to the realistic constellation encoder and decoder implementation requirements, for easy modulation in the practical bit filling procedure, the allocated bit number for each channel should be an integer. A suboptimal integer bit loading algorithm is given in the following section.

\section{Suboptimal Integer Bit Loading Algorithm}

The transmitted bits $\left(b_{i, j}\right)$ of ST $i$ on channel $j$ according to $P_{i, j}$ can be rewritten as in (26) according to (7).

$$
b_{i, j}=\log _{2}\left(1+P_{i, j} N_{i, j}\right)
$$

According to the power filling level on each channel, obtained from Algorithm 1 and Algorithm 2, if we increase all the bit rates to the upper bound of optimal transmission bits $x\left(\left[b_{i, j}\right\rceil\right)$, which is the nearest integer that is larger than $x$, not only will the power budget be exceeded, but also the probability of unaffordable interference to primary receivers will increase. However, a reliable integral data rate can be achieved if the transmitted bits $b_{i, j}$ are uniformly reduced to the lower boundary $\left(\left\lfloor b_{i, j}\right\rfloor\right)$, in which $\lfloor x\rfloor$ is the lower bound of $x$ that takes the nearest smaller integer than $x$. We define the extra power that is needed to transmit bits on channel $\mathrm{j}\left(j \in C_{i}, i=1,2,3, \ldots, K\right)$ as $P_{i, j}^{\mathrm{inc}}$ in (27).

$$
P_{i, j}^{\text {inc }}=N_{i, j}\left(2^{b_{i, j}}-1\right)-N_{i, j}\left(2^{\left\lceil b_{i, j}\right\rceil}-1\right)
$$

Then, $P_{C_{i}}^{\text {tot }}$, which is defined as the total saved power of ST $i(i=1,2,3 \ldots, K)$ on the selected channel set $C_{i}\left(C_{i} \in N\right)$ when we perform only the low bound integer bit loading, is given in (28).

$$
P_{C_{i}}^{t o t}=\sum_{j \in C_{i}}\left[N_{i, j}\left(2^{b_{i, j}}-1\right)-N_{i, j}\left(2^{\left\lfloor b_{i, j}\right\rfloor}-1\right)\right]
$$

Clearly, as we can see from (28), even though the integer bit loading is able to protect primary receivers, the lower bound of allocated bits on each channel will cause a waste of the power budget. Therefore, we try to transmit $\lceil x\rceil$ bits on channel set $C_{i}$ as long as such increment does not exceed the power budget $P_{\text {budget }}$, while obeying the PSM constraint of $\mathrm{C}_{\mathrm{i}}$ at the same time. To fully utilize the power budget and maximize the transmission data rate, a suboptimal (SubOpt) integer bit loading algorithm is proposed in Algorithm 3 (Figure 10).

Input : allocated bits $b_{i, j}$ on channel set $C_{i}$

Output: SubOpt bit loading

$$
\begin{aligned}
& \text { for } j=0, j<\left|C_{i}\right|, j++ \\
& \quad P_{i, j}^{\prime}=N_{i, j}\left(2^{\left\lceil b_{i, j}\right\rceil}-1\right) \\
& \text { if } P_{C_{i}}^{\text {tot }}+P_{j}^{\text {inc }} \geq 0 \quad \text { \&\& } P_{i, j}^{\prime} \leq P_{i, j}^{\text {Max }} \text { then } \\
& \text { continue } \\
& \text { else } \\
& \quad P_{i, j}^{\prime}=N_{i, j}\left(2^{\left\lfloor b_{i, j}\right\rfloor}-1\right) \\
& \text { endif } \\
& \text { calculate } b_{i, j} \text { by (25) with } P_{i, j}^{\prime} \\
& P_{C_{i}}^{\text {tot }}=P_{C_{i}}^{\text {tot }}+P_{j}^{i n c}
\end{aligned}
$$

Figure 10 Suboptimal Bit Loading Algorithm (Algorithm 3). 
After the suboptimal integer bit loading, the remaining power budget is less than the power needed to transmit one more bit on any channel $j\left(j \in C_{i}\right)$. Therefore, this is a nearly optimal solution to the integral bit loading requirement.

\section{Simulation results}

In this section, simulations are conducted to evaluate the performance in terms of: i) interference avoidance performance during data transmission; ii) power loading model and corresponding capacity maximization algorithms; and iii) SubOpt IBL loading. A typical waterfilling scheme [10] is compared as a conventional protocol, in which the PSM constraint is estimated based only on its local primary sensing information without the coordination of neighbors. The simulation parameters are shown in Table 2. The Gaussian noise is assumed to have zero mean and unit variation. Other power related energies are defined in decibels compared with noise power.

Figure 11 shows the simulation topology for the proposed DNPSM and the reliable capacity maximization model. Without loss of generality, we assume that a total of three worst situation primary receivers ( $\mathrm{PRa}, \mathrm{PRb}$ and PRc) on sub-channel sets A (Ch1-Ch5), B (Ch6-Ch10), and $\mathrm{C}$ (Ch11-Ch15) exist nearby, and that each PR is placed on the primary signal decodable boundaries of its corresponding PT. Two STs exist and compete with each other to choose the most suitable channel set from $(\mathrm{A}, \mathrm{B}, \mathrm{C})$ based on the comparison of $\arg \underset{i \in K}{\operatorname{Max}}\left(\arg \underset{j \in N}{\operatorname{Min}}\left(D_{\mathrm{ST} i, \mathrm{PR}}^{j}\right)\right)$; these STs then pour the power with obtained optimal power filling amount $P_{i, j}^{\mathrm{Opt}}\left(P_{a, j}^{\mathrm{Opt}}\right.$ and $P_{b, j}^{\mathrm{Opt}}$ in Figure 11) from (20) into the selected channel set $C_{i}\left(C_{a}\right.$ and $C_{b}$ in this case), respectively. The DNPSM performed by the secondary transmitter STa in order to avoid the harmful interference for the worst situation of PRa is picked up as an example to illustrate the performance of the DNPSM. Secondary neighbors are randomly placed within the transmission range of the ST. Only SNs that are within the signal overlapping area between the PT and ST can help the ST to derive the transmission power related safety distance in the DNPSM. Data are always backlogged at the ST side.
As can be seen in Figure 11, to evaluate the performance of the proposed method, DNPSM, and in accordance with the different positions of the $S T$, we use a coexistence scenario between PTa and STa and place STa between $\left(x_{\mathrm{PR}}+0,0\right)$ and $\left(x_{\mathrm{PR}}+T R_{\mathrm{ST}}^{\mathrm{Max}}, 0\right)$, in which $x_{\mathrm{PR}}=150 \mathrm{~m}$ is the PRa's position on the $\mathrm{x}$-axis. Since the performance of the DNPSM mainly depends on the variation of the position of the ST ( $\left.D_{\mathrm{ST}, \mathrm{PR}}\right)$, on the transmission range of the ST (ST_Radius) on the CR control band, and on the number of neighbors, we changed these parameters in this simulation study. The appearance of the primary signal is based on the On/Off mode. During On-time, the channel is busy. On-time $\left(T_{\text {On }}\right)$ and Off-time $\left(T_{\text {Off }}\right)$ have exponential distributions $\lambda_{1}$ and $\lambda_{2}$, respectively, as expressed in (28). Here, the primary system manifestation probability $P_{\text {On }}$ is calculated using (30).

$$
\begin{aligned}
& F_{\text {On }}(t)=P\left(T_{\text {On }} \leq t\right)=1-e^{-\lambda_{1} t} \\
& F_{\text {Off }}(t)=P\left(T_{\text {Off }} \leq t\right)=1-e^{-\lambda_{2} t} \\
& P_{\text {On }}=\frac{E\left(T_{\text {On }}\right)}{E\left(T_{\text {On }}\right)+E\left(T_{\text {Off }}\right)}=\frac{1 / \lambda_{1}}{1 / \lambda_{1}+1 / \lambda_{1}}=\frac{\lambda_{1} \lambda_{2}}{\lambda_{1}\left(\lambda_{1}+\lambda_{2}\right)}
\end{aligned}
$$

Figure 12(a) shows the interference ratio in accordance with the different number of SNs. Interference ratio (31) indicates how much interference on average the surrounding primary systems experienced during the whole CR transmission time. In this experiment, in order to obtain a fair parameter comparison value to evaluate the system performance, the transmission radius (ST_Radius) is set at 230 meters and the appearance probability of the primary signal on the licensed band is set at $50 \% . T_{d_{i}}$ is the $i$-th data transmission time, $N_{t}$ is the total number of data transmissions, and $I T_{j}$ is the interference time for the primary system of the $j$-th data transmission time. In the case of the conventional scheme, regardless of the number of SNs, a CR communication pair cannot obtain the correct measurement due to the lack of a neighbor cooperated power controlling mechanism, so it always yields heavy interference to the incumbent systems around it. In the proposed DNPSM scheme, as the number of SNs increases, the interference ratio decreases dramatically. Compared

\begin{tabular}{|c|c|c|c|}
\hline Parameter & Value & Parameter & Value \\
\hline Number of SNs & $5 \sim 90$ & $P_{\mathrm{PT}}(\mathrm{PT}$ transmission power $)$ & $320 \mathrm{~dB}$ on each sub-channel \\
\hline$D_{\mathrm{PR} a, \mathrm{ST} b}$ & $220 \mathrm{~m}$ & $D_{\text {PRa,STa }}$ & $300 \mathrm{~m}$ \\
\hline$D_{\mathrm{PR} b, \mathrm{ST} b}$ & $225 \mathrm{~m}$ & $D_{\mathrm{PR} b, \mathrm{ST} a}$ & $80 \mathrm{~m}$ \\
\hline$D_{\text {PRC,STb }}$ & $150 \mathrm{~m}$ & $D_{\mathrm{PR} C, \mathrm{ST} a}$ & $260 \mathrm{~m}$ \\
\hline $\mathrm{TR}_{\mathrm{PT}}$ & $5 \mathrm{~km}$ & ST transmission power (TP ST $_{\text {MT }}$ ) & $170 \mathrm{~dB}$ on each sub-channel \\
\hline
\end{tabular}
with the conventional PSM constraint estimation scheme,

Table 2 Simulation parameters 


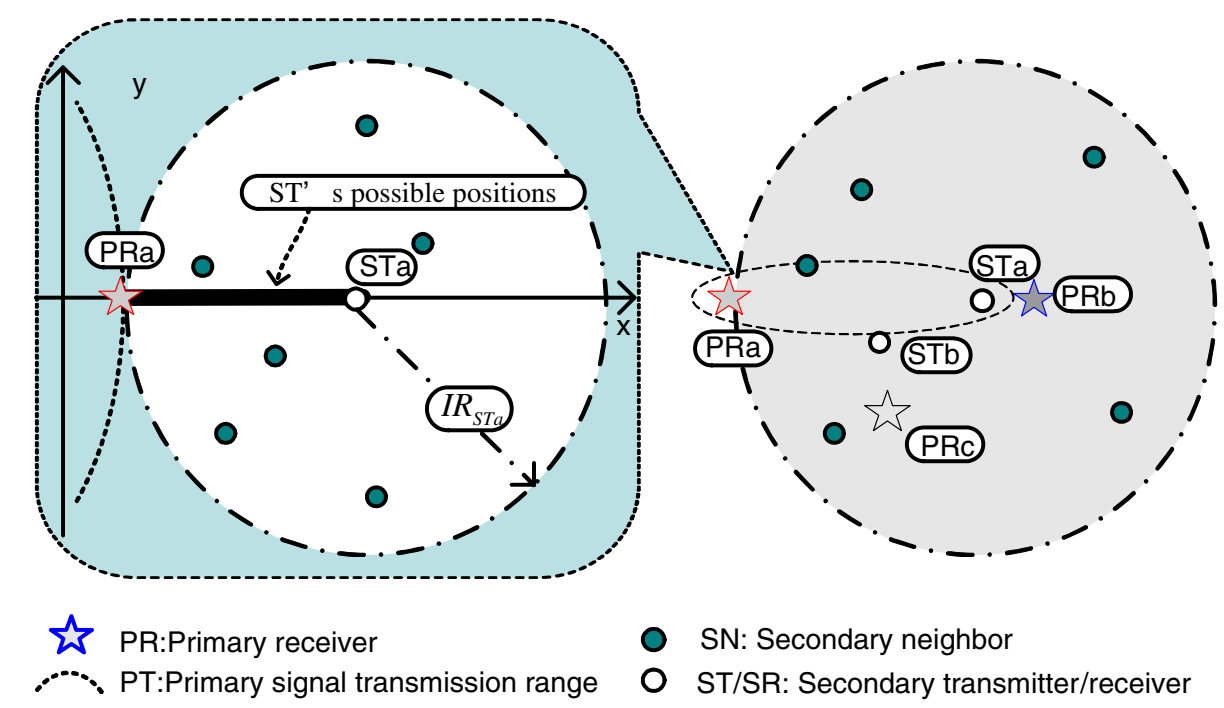

Figure 11 Simulation topology on licensed/CR control band.

when the nodes' density is high enough, harmful interference to the primary system can be completely avoided. Since the smaller overlapping area between ST and PT transmission ranges results in fewer existing $\mathrm{SNs}$, the larger safety distance $\left(D_{\mathrm{ST}, \mathrm{PR}}\right)$ always has a higher interference ratio. The variation of the interference ratio according to the transmission range of the ST on the CR control band (ST_Radius) is also shown in Figure 12(b). We can see that only 20 randomly distributed SNs are enough to lower harmful interference to under $2 \%$.

$$
\text { Inference Ratio }=\sum_{j=1}^{N_{t}} I T_{j} / \sum_{i=1}^{N_{t}} T_{d_{-} i}
$$

Due to the random position of the SNs, there is a little possibility that no SNs exists on the straight line between the ST and the PT. When an SN that is not located on the straight line between the ST and the PT overhears the control information (channel sensing request) from the ST, the distance estimated $\left(D_{S T . P R}\right)$ by this $\mathrm{SN}$ will be a little bit longer than the real distance between the ST and the possible hidden PR. This discrepancy can cause extra interference to the primary devices. Since precise distance estimation is important to provide accurate power control for SUs, we observed the distance estimation error and the transmission power reduction ratio, with data shown in Figure 13. The distance estimation error represents the difference between the real distance $R D_{\text {ST,PR }}$ and the final estimated distance $D_{S T, P R}$, which is obtained by the ST. As can be seen in Figure 13(a), at first, when $D_{S T, P R}$ increases, the overlapping area decreases and the number of neighbor nodes that can

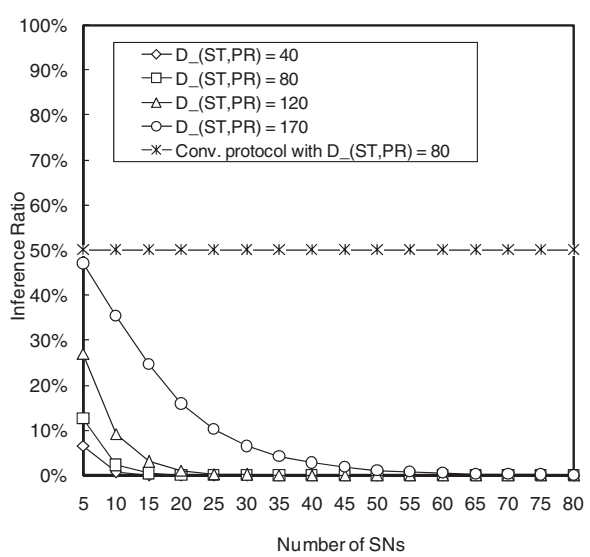

(a) Inference Ratio with Number of SNs variation.

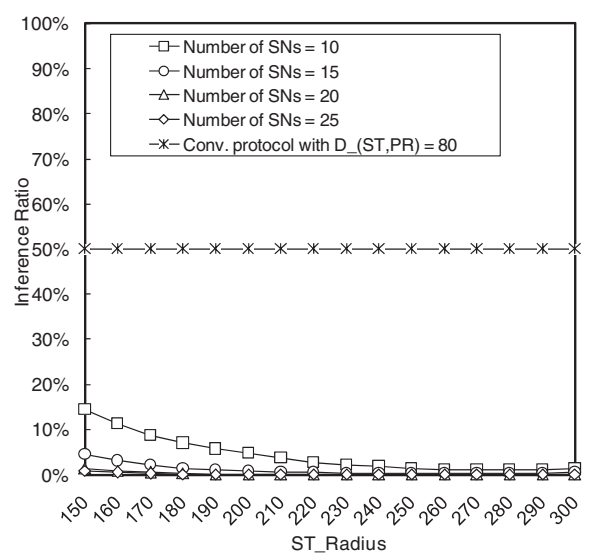

(b) Inference Ratio with ST_Radius variation

Figure 12 Average interference ratio to primary system. 


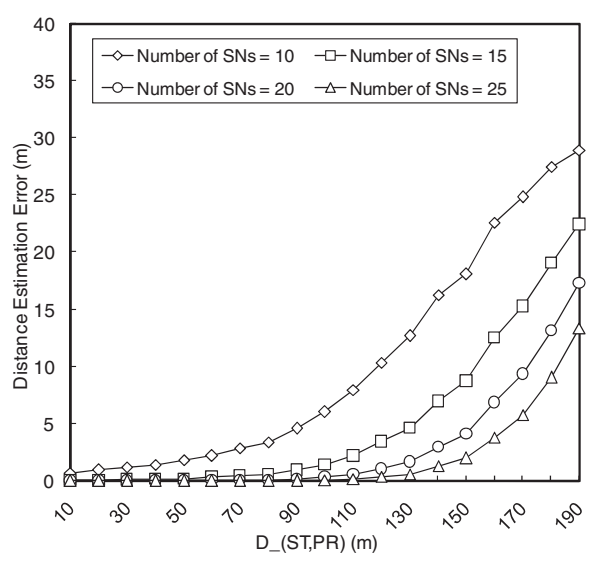

(a) Distance estimation Error

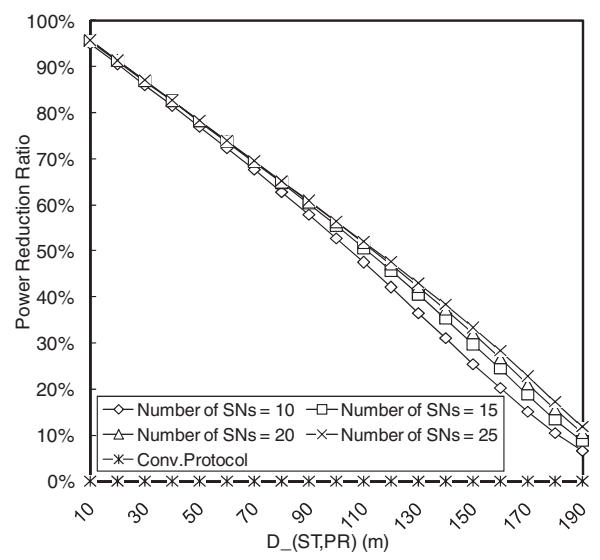

(b) Power reduction ratio

Figure 13 Distance estimation error \& power reduction ratio of the proposed DNPSM.

detect the primary signal also decreases; the distance estimation error increases consequently. However, since the distance estimation error is minor compared with the estimated safety distance, the number of SNs has little effect on the power reduction ratio. Figure 13(b) illustrates the relationship between the average reduced transmission power ratio and the positions of the ST. As the safety distance increases, the power reduction ratio of the conventional PSM estimation remains the same. Due to incorrect incumbent system sensing results, the conventional PSM scheme always uses the full power to transmit, which will cause harmful interference to the incumbent systems. Obviously, the DNPSM has better performance. When $D_{\mathrm{ST}, \mathrm{PR}}$ equals $90 \mathrm{~m}$, the number of $\mathrm{SNs}$ equals 15 and the ST_Radius is $150 \mathrm{~m}$; in such a case, less than $50 \%$ of the full power level will be allowed for secondary data transmission, in order to avoid harmful interference to possible PRs that are around.
The effect of the proposed PSM constraint derived from the DNPSM on the power loading (Water-Filling) performance (Pro. WF) is shown in Figure 14. Without loss of generality, we chose Algorithm 2 to illustrate the power loading performance on the selected subchannels, with different power loading bottoms and PSM constraints shown in Figure 11. To clearly show the effect of the DNPSM on the performance of the proposed water-filling (Pro.WF) scheme, we set the overall power budget ( $P_{\text {budget }}$ ) equal to $920 \mathrm{~dB}$. As shown in Figure 14, compared with the conventional WF scheme (Conv. WF), the maximal allowed transmission power will be smaller with cooperation from one-hop neighbors (PSM constraint (14)). According to the channel selection criteria ( $\arg \underset{i \in K}{\operatorname{Max}}\left(\underset{j \in N}{\arg \operatorname{Min}}\left(D_{S T i, P R}^{j}\right)\right)$ ), STa will choose sub-channel set A (Ch1-Ch5) and C (Ch11-Ch15) as the channels into which to pour power (Figure 14(a)), while

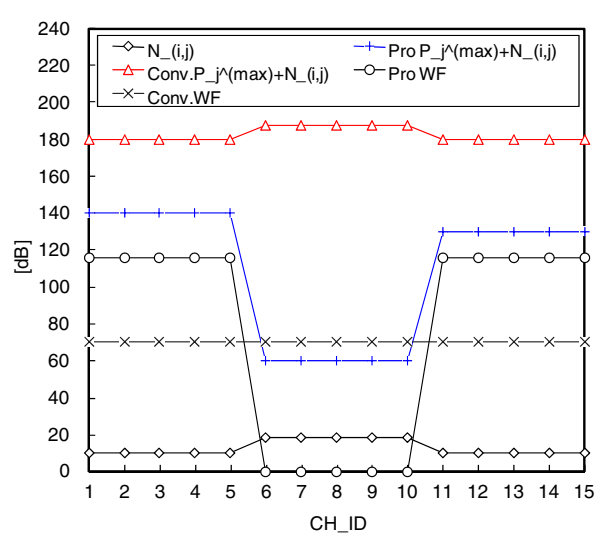

(a) Power loading on STa

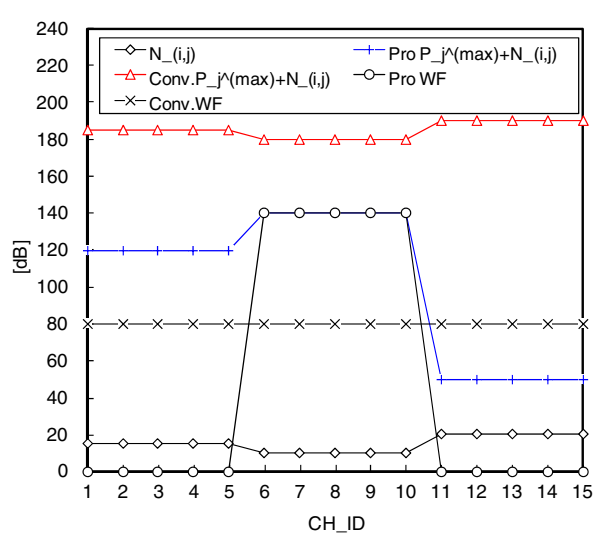

(b) Power loading on STb

Figure 14 Power loading illustration according to channel selection and PSM constraint. 
STb will choose channel set B (Ch6-Ch10) (Figure 14(b)). On channel set B (Ch6-Ch10), though STb has a large enough power budget to allow it to pour more water, it still uses the $P_{b, j}^{\mathrm{Max}}\left(j \in C_{B}\right)$ as the optimal water level to protect $\mathrm{PRb}$. Compared with the proposed DNPSM scheme, the conventional approach cannot detect the existence of primary signals on channel sets $\mathrm{A}, \mathrm{B}$, or $\mathrm{C}$, and so it always uses the allowed hardware maximal transmission power $\left(\mathrm{TP}_{\mathrm{ST}}^{\mathrm{Max}}\right.$ ) as the PSM constraint to pour power into all the sub channels. Harmful interference is given to PRs on those channels (PRs working on channel set B (Ch6-Ch10) and C (Ch11-Ch15) in Figure 14(a) and Figure 14(b), respectively).

In Figure 15, the Pro. WF (Algorithm 2) at the STa side is selected as an example to show the effect of integer bit loading on the power loading of each channel set with different $P_{a, j}^{\mathrm{Max}}\left(j \in C_{A}, C_{B}, C_{C}\right)$. Since significant harmful interference has already been introduced by using the conventional water-filling schemes (Conv. WF), we will not consider the SubOpt bit loading performance for Conv. WF. As that is shown in Figure 15(a), the lower band integer bit loading (Pro. WF w. IBL) will give a different level of power reduction. However, compared with Conv. WF, which does not take the pure primary receiver into account (performing the data transmission on channel set B ((Ch6-Ch10)), the proposed distributed neighbor coordinated water-filling mechanism (Pro. WF) not only gives reliable protection to the primary users, but also enhances the feasible transmission rate (channel set $\mathrm{A}$ and channel set C). Clearly, as we can see in Figure $15(\mathrm{~b})$, the longer safety distance $P_{a, j}^{\mathrm{Max}}(j \in$ $\left.C_{A}, C_{B}, C_{C}\right)$ ) will have a higher accumulated transmission bit rate $\left(C_{A}\left(\right.\right.$ Ch1-Ch5) $>C_{C}($ Ch11-Ch15) $)$, because the maximal transmission power can be reached earlier by increasing the transmission power (enhancing the transmission rate) on a lower $\left.P_{a, j}^{\mathrm{Max}}\left(j \in C_{A}, C_{B}, C_{C}\right)\right)$ channel.

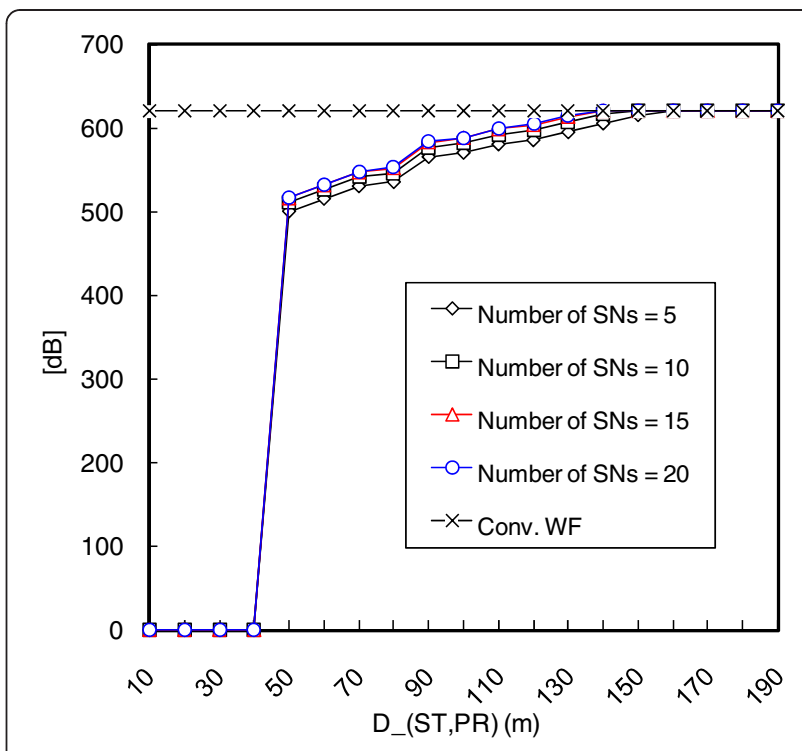

Figure 16 Performance of proposed PSM constraint on power loading.

Figure 16 provides an evaluation of the proposed power loading performance according to the different numbers of randomly placed SNs with different positions of the ST. Along with an increasing $D_{S T, P R}$, the total amount of pouring power also increases, because the maximal allowed transmission power $\left(P_{i, j}^{\mathrm{Max}}\right)$ has been increased. However, when $D_{S T, P R}$ increases to a certain level (ST_Radius $=200 \mathrm{~m}$ ), the total amount of power will stop the increasing trend and stay at the same level due to power budget limitations. Furthermore, as we increase the number of SNs, the total amount of power will be slightly reduced. The reason for this is that the larger number of SNs will reduce the safety distance estimation error (tightening the PSM constraint), which will result in a smaller amount of pouring water (power). When the number of SNs is close to 20, as we can see in Figure 12,

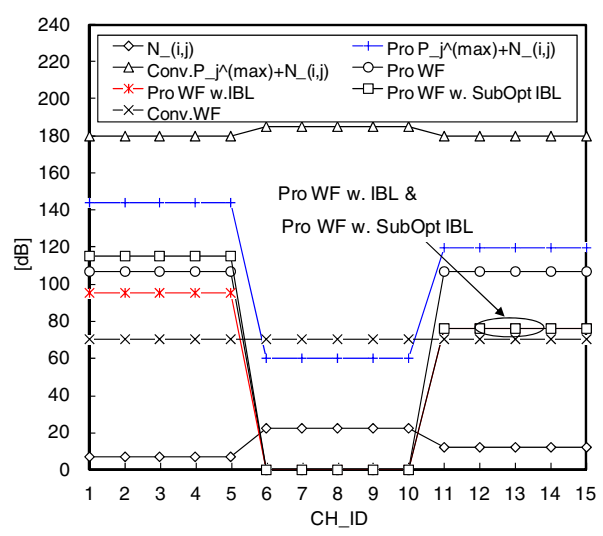

(a) WF variation according to the integer bit loading.

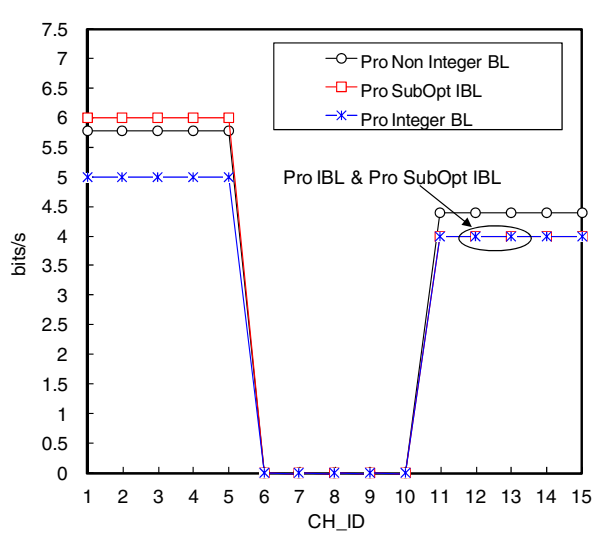

(b) Bit loading illustration

Figure 15 Performance of Integer bit loading algorithm. 
in which the distance estimation error is less than $2 \%$, minor power pouring changes are made.

Figure 17 shows the data rate sum of the CR transmission system achieved by performing Algorithm 1 and Algorithm 2 separately, with implementation of Algorithm 3. Using Algorithm 2, STa chooses channel set A and C, while STb chooses just channel set B according to the channel selection criteria under the situation shown in Figure 11. The average rate (Ave. Rate), which is obtained by performing Algorithm 1, denotes the average of the transmission rate sum determined by applying the channel selection without neighbor coordination at STa and STb. That is, STa or STb chooses all available channels in order to perform power loading without considering the channel selection strategy. Rate with NC denotes the rate sum that results by applying the channel selection strategy with neighbor coordination (Algorithm 2). Because each channel has a different power loading level, and uniform lower bound integer bits loading (IBL) causes an uneven transmission power waste, Algorithm 2 captures this difference and determines the optimal channel selection strategy to reduce power budget waste by reassigning the suitable sub-channels to each CR communication pair. As we can see in Figure 17, compared to the power loading of Algorithm 1, which is used to maximize the system throughput, Algorithm 2 efficiently enhances the overall transmission rate while reducing the power budget waste. This also indicates that in order to maximize the system throughput, the ST prefers to allocate more power to a channel that is far away from the primary receivers. Along with the increasing of the Power_budget, the data transmission rate sums of both channel selections (with or without an optimal channel selection strategy) show an ascending trend. However,

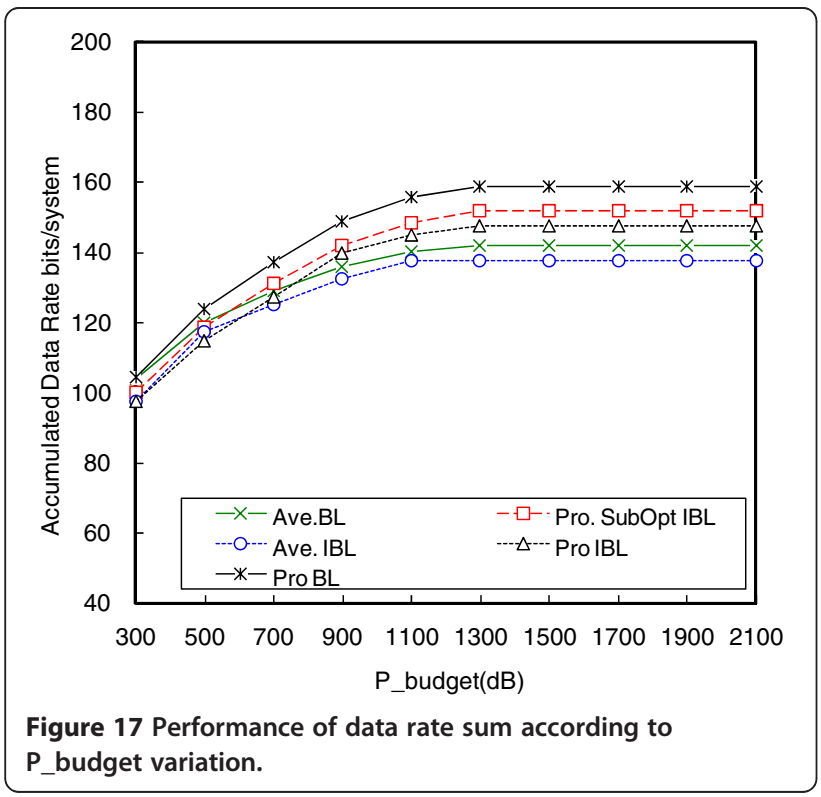

when the Power_budget reaches a certain level, due to the PSM constraint (14), the data transmission rate sums of both channel selections stop the continuous increasing trend. Obviously, with the SubOpt IBL algorithm (Algorithm 3), the CR system capacity can be further enhanced.

As we can see from the evaluations above, compared with the conventional power loading scheme, which does not consider the pure primary receiver, the proposed scheme provides reliable protection for primary users. With the proposed simple but efficient iterative power loading algorithms, a suitable channel set can be determined and optimal transmission power on each channel for whole system capacity maximization can be achieved successfully.

\section{Conclusion}

In this paper, a neighbor coordination capacity maximization model with a reliable interference free constraint is proposed for cognitive radio ad hoc networks with multiple secondary nodes; in this model, the complex mutual interference relationship between primary users and secondary users is modeled as an optimization problem and the reliability of the interference free constraint during the Lagrange derivation procedure can be guaranteed. To derive a reliable PSM constraint, instead of using local sensing to estimate the distance to a possible primary receiver to avoid harmful interference, the secondary transmitter estimates the distance to the hidden primary receiver using neighbor node cooperation, and the transmission power is adaptively controlled by considering the safety distance. We jointly considered the relationship among channel set selection, total transmission power budget, and PSM limitation, and proposed two simple but efficient iterative power allocation algorithms, along with a suboptimal integer bit loading algorithm, in which both distributed and neighbor cooperative comparison based channel selection strategies are considered. Simulation results show that with the help of the proposed PSM constraint estimation method and efficient power loading algorithms, capacity maximization of the secondary system, along with reliable performance of the primary user without harmful interference, can be successfully achieved.

\section{Competing interests}

The authors declare that they have no competing interests.

\section{Authors' information}

Graduate School of Information Technology \& Telecommunication, Inha University, 253 Yonghyun-dong, Nam-gu, Incheon 402-751, Korea, http:// multinet.inha.ac.kr 


\section{Author details}

'Alcatel-Lucent Shanghai Bell, 388, Ningqiao Road, JinqiaoPudongShanghai 201206, P.R. China. ${ }^{2}$ Graduate School of Information Technology \& Telecommunication, Inha University, 253 Yonghyun-dong, Incheon, Nam-gu 402-751, Korea.

Received: 20 December 2011 Accepted: 19 June 2012

Published: 18 September 2012

\section{References}

1. FCC, Spectrum Policy Task Force Report, No.02-155 (2002)

2. H. Simon, Cognitive Radio: Brain-Empowered Wireless Communications. IEEE Journal on Selected Areas in Communications 23(2), 201-220 (2005). doi: 10.1109/JSAC.2004.839380

3. Q. Zhao, L. Tong, A. Swami, Decentralized Cognitive MAC for Dynamic Spectrum Access (the IEEE Symposium on New Frontier in Dynamic Spectrum Access Networks (DySPAN'05), Baltimore, Maryland, USA, 2005)

4. Q. Zhao, L. Tong, A. Swami, Y. Chen, Decentralized cognitive MAC for opportunistic spectrum access in Ad Hoc networks: a POMDP framework. IEEE Journal on Selected Areas in Communications 25(3), 589-600 (2007). doi:10.1109/JSAC.2007.070409

5. H. Alex Chia-Chun, S. David, L. Wei, C.-C. Jay Kuo, A Cognitive MAC Protocol Using Statistical Channel Allocation for Wireless Ad-hoc Networks (IEEE Wireless Communications Networking Conference (WCNC'07), Hong Kong, China, 2007), pp. 11-15

6. F. Chu, K. Chen, Radio Resource Allocation in OFDMA Cognitive Radio Systems (the IEEE Personal, Indoor and Mobile Radio Communications Symposium (PIMRC' 07), Athens, Greece, 2007), pp. 3-7

7. J.-A. Bazerque, G.B. Giannakis, Distributed scheduling and resource allocation for cognitive OFDMA radios (the IEEE CrownCom 2007, Orlando, 2007), pp. 1-3

8. A. Attar, O. Holland, M. Nakhai, A. Aghvami, Interference-limited resource allocation for cognitive radio in orthogonal frequency-division multiplexing networks. IET Communications 2, 806-814 (2008). doi:10.1049/ iet-com:20070355

9. K. Hamdi, Z. Wei, K. Letaief, Uplink scheduling with QoS provisioning for cognitive radio systems (the IEEE Wireless Communications Networking Conference (WCNC'07), Hong Kong, China, 2007), pp. 11-15

10. K. Xin, L. Ying Chang, N. Arumugam, Optimal power allocation for fading channels in Cognitive Radio Networks under Transmit and Interference Power Constraints (the IEEE International Conference on Communications 2007(ICC 2007), Glasgow, Scotland, 2007), pp. 24-28

11. S. Kyuho, J. Bang Chul, C. Song, S. Dan Keun, Opportunistic Underlay Transmission Multi-carrier Cognitive Radio Systems (the IEEE Wireless Communications Networking Conference 2009 (WCNC'09), Budapest, Hungary, 2009), pp. 5-8

12. P. Wang, M. Zhao, L. Xiao, S. Zhou, J. Wang, Power allocation in OFDM-based cognitive radio systems (the IEEE Global Communications Conference 2007 (IEEE GlobeCom 2007), Washington DC, USA, 2007), pp. 26-30

13. M. Yao, K. Dong In, Centralized and distributed optimization of ad hoc cognitive radio network (the IEEE Global Communications Conference 2009 (IEEE GlobeCom 2009), Hilton Hawaiian Village, Hawaii, USA, 2009) pp. 1-7

14. M. Yao, K. Dong In, Sub-channel-sharing based distributed optimization of ad hoc cognitive radio network (the IEEE Global Communications Conference 2010 (IEEE GlobeCom 2010), Miami, Florida, USA, 2010) pp. 1-6

15. Z. Cheng Shi, K. Kyungsup, Power/Bit loading in OFDM based cognitive networks with comprehensive interference considerations: the single SU case. IEEE Trans. Vehicular Technology 59(4), 1910-1922 (2010). doi: 10.1109/TVT.2010.2042091

16. IEEE 802.22-2011(TM) Standard for Cognitive Wireless Regional Area Networks (RAN) for Operation in TV Bands (2011)
17. A. Sahai, N. Hoven, R. Tandra, Some fundamental limits on cognitive radio (the Allerton Conference on Communication, Control, and computing, University of Illinois, 2004) pp. 1-11

18. M. Hata, Empirical formula for propagation loss in land mobile radio services. IEEE Trans. Vehicular Technology VT-29, 317-325 (1980). doi:10.1109/T-VT.1980.23859

19. Digital Mobile Radio towards Future Generation Systems, Digital Mobile Radio towards Future Generation Systems (COST 231, Brussels, 1996)

20. D.P. Palomar, J.R. Fonollosa, Practical algorithms for a family of waterfilling solutions. IEEE Trans. Signal Processing 53(2), 686-695 (2005). doi:10.1109/ TSP.2004.840816

21. S. Boyd, L. Vandenberghe, Convex Optimization (Cambridge Univ. Press, Cambridge. U.K, 2004)

doi:10.1186/1687-1499-2012-295

Cite this article as: Hao and Yoo: Interference avoidance throughput optimization in cognitive radio ad hoc networks. EURASIP Journal on Wireless Communications and Networking 2012 2012:295.

\section{Submit your manuscript to a SpringerOpen ${ }^{\odot}$ journal and benefit from:}

- Convenient online submission

- Rigorous peer review

- Immediate publication on acceptance

- Open access: articles freely available online

- High visibility within the field

- Retaining the copyright to your article

Submit your next manuscript at springeropen.com 